\title{
OSTEOARTROSIS EN CAZADORES-RECOLECTORES DE PATAGONIA CENTRO-MERIDIONAL (SANTA CRUZ, ARGENTINA) DURANTE EL HOLOCENO TARDÍO: UNA APROXIMACIÓN A LOS PATRONES DE ACTIVIDAD
}

\author{
OSTEOARTHROSIS IN HUNTER-GATHERERS OF CENTRAL-SOUTHERN \\ PATAGONIA (SANTA CRUZ, ARGENTINA) DURING THE LATE HOLOCENE: AN \\ APPROXIMATION TO THE ACTIVITY PATTERNS
}

\author{
Milena Constanza Morlesín ${ }^{1}$ y Solana García Guraieb $b^{1,2}$
}

\begin{abstract}
La osteoartrosis (OA) es la modificación patológica más comúnmente hallada en restos óseos humanos. Si bien existe una multiplicidad de factores causales de dicha modificación, la actividad física repetida genera cambios articulares. Por tal motivo, ha sido estudiada desde una perspectiva bioarqueológica para abordar el estudio de los patrones de actividad de poblaciones del pasado. Este trabajo busca analizar las modificaciones óseas de la columna vertebral y de las articulaciones apendiculares en una muestra de 14 individuos del Holoceno Tardío recuperados en la cuenca del Lago Salitroso (Santa Cruz, Argentina). Los objetivos son: (a) evaluar la paleoepidemiología de la OA y su relación con otras artropatías (p.ej., nódulos de Schmorl), y (b) vincular la relación entre los patrones de variación de la OA con aspectos fisiológicos como el sexo y la edad. Esta información permitirá contribuir al conocimiento de los comportamientos humanos vinculados al uso del cuerpo y a las actividades realizadas, en el marco de las fluctuaciones climáticas y los cambios organizativos propuestos para esta población durante el Holoceno Tardío. Todos los individuos presentan OA, hallándose escasas diferencias entre los sexos, y una mayor frecuencia de OA en individuos mayores a 40 años, y en aquellos datados entre el ca. 800 y 350 años AP.

Palabras claves: artropatías, uso del cuerpo, Lago Salitroso, poblaciones patagónicas, Holoceno Tardío.
\end{abstract}

Osteoarthrosis (OA), a condition caused, among many other factors, by repeated physical activity, is the most commonly found pathological bone modification in human skeletal remains. Accordingly, it has been frequently studied from a bioarchaeological perspective, in order to identify the patterns of activity of populations that preceded us. This study aims to analyze the bone modifications in the spine and the appendicular joints of 14 adult individuals of both sexes from the Late Holocene found in the Salitroso Lake basin (Santa Cruz, Argentina). The objectives are (a) to evaluate the paleoepidemiology patterns and its relationship with other arthropathies (e.g. Schmorl's nodes); and (b) to explore the connection between OA variation patterns and aspects such as sex and age. This information will contribute to the knowledge of human behavior regarding body use and performed activities within the context of the climatic shifts and organizational changes that affected this population during the late Holocene. The data reveal that, with few differences between sexes, all the individuals exhibit OA and that the prevalence of $O A$ is higher in older individuals and in those whose chronology is between ca. 800 and 350 years BP.

Key words: Arthropathies, body use, Salitroso Lake, Patagonic human population, Late Holocene.

La osteoartrosis $(\mathrm{OA})$, también conocida como enfermedad degenerativa articular (EDA) ${ }^{1}$, es una enfermedad progresiva, no necesariamente ligada a un proceso inflamatorio de origen, que produce la alteración del cartílago articular y del hueso subcondral de las articulaciones sinoviales (Aufderheide y RodríguezMartín 1998; Burt et al. 2013; Jurmain 1991; Lieverse et al. 2007; Ortner 2003; Weiss y Jurmain 2007). Presenta una etiología compleja debido a la multiplicidad de factores que inician o interactúan en la cadena de eventos que deriva en la afección de la articulación (Arden y Nevitt 2006; Hunter 2011; Jurmain 1977; Rogers y Waldron 1995; Waldron 2009). En este sentido, la edad, el sexo, la predisposición genética, las influencias anatómicas, traumas, entre otros, pueden influir en la manifestación de la OA (Bridges 1992;

\footnotetext{
${ }^{1}$ Consejo Nacional de Investigaciones Científicas y Técnicas (CONICET) - Instituto Nacional de Antropología y Pensamiento Latinoamericano. Buenos Aires, Argentina.mile.morlesín@gmail.com; solanagg@gmail.com

${ }^{2}$ Universidad de Buenos Aires, Facultad de Filosofía y Letras, Buenos Aires, Argentina.
} 
Burt et al. 2013; Jurmain 1977; Ortner 2003; Rogers et al. 1987; Waldron 2009; Zhang y Jordan 2010). A pesar de que generalmente se asocia la OA con la edad avanzada, no es la edad en sí misma, sino el carácter acumulativo del desgaste fisiológico de las articulaciones, resultado del uso intenso y repetido de las mismas, el desencadenante de esta condición (Jurmain 1990; Lieverse et al. 2007). De hecho, se ha observado una correlación entre altas frecuencias y grados de severidad de la osteoartrosis y poblaciones humanas con modos de vida físicamente demandantes (Jurmain 1977, 1980; Kennedy 1989; Larsen 1997; Weiss y Jurmain 2007). Por tal motivo, teniendo en cuenta y controlando - cuando la información disponible lo permita- los otros factores que pueden contribuir a la manifestación de la OA, consideramos que es un hecho reconocido que la actividad física repetida genera cambios articulares y, por ende, juega un papel importante en la expresión de la OA, especialmente en determinadas articulaciones (hombro, codo, cadera y rodilla) (Bridges 1991, 1993; Burt et al. 2013; Jurmain 1977, 1980, 1990; Larsen 1997; Weiss y Jurmain 2007) y superados determinados umbrales de intensidad. Por ello, el estudio de su frecuencia y distribución en las poblaciones humanas del pasado puede contribuir, no solo a aumentar nuestra comprensión y conocimiento paleoepidemiológico de dicha condición, sino también aportar información relevante para discutir hipótesis relacionadas con el uso del cuerpo y los patrones de actividad de los grupos humanos y sus cambios a través del tiempo.

Las investigaciones arqueológicas realizadas en Patagonia Centro-meridional (Figura 1) están orientadas a conocer los patrones de movilidad y uso del espacio

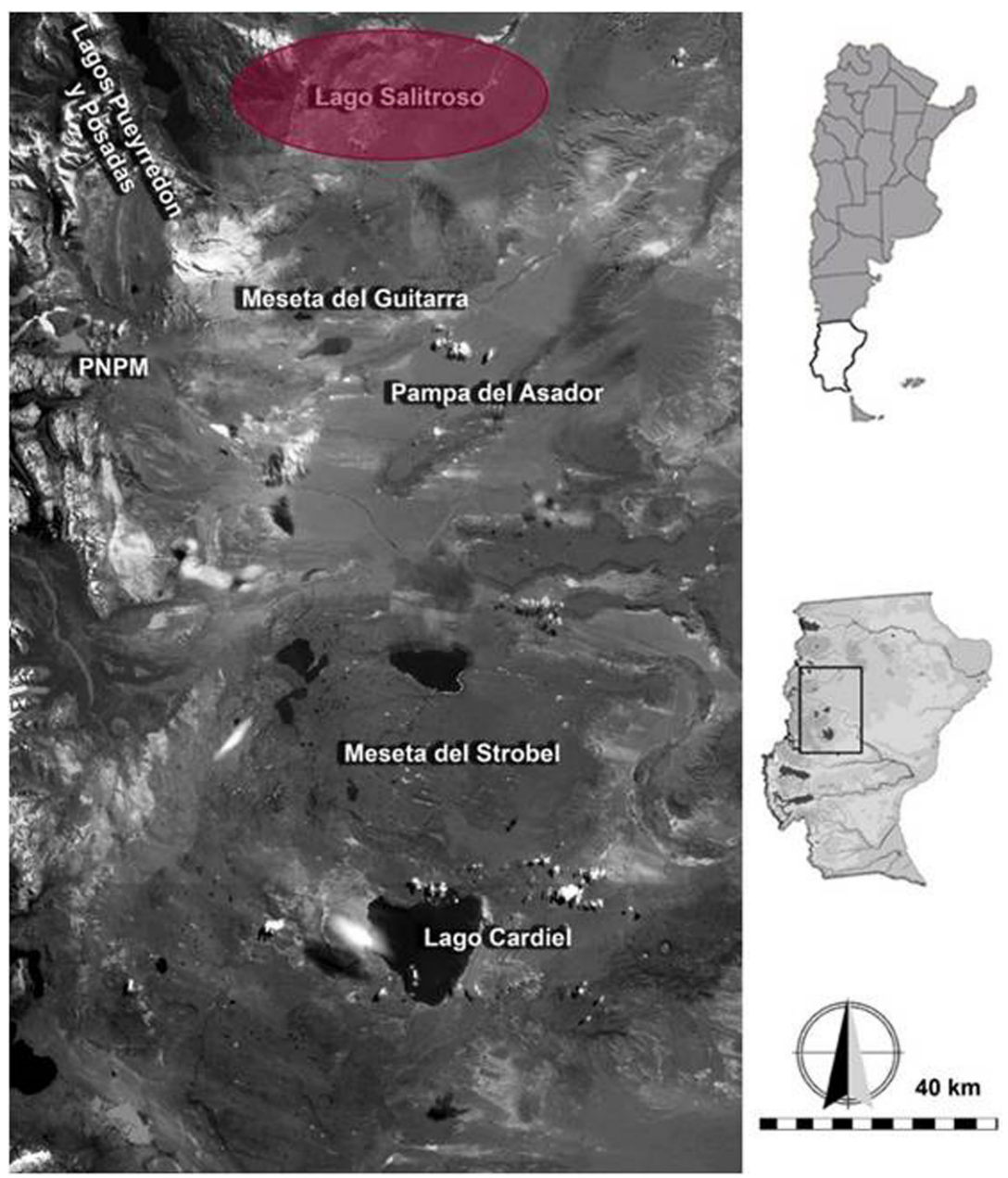

Figura 1. Mapa de la delimitación geográfica de la región de estudio. En violeta se destaca el área particular de estudio que corresponde a la cuenca del Lago Salitroso.

Map of the geographical delimitation of the study region. In violet, the particular study area of the Salitroso Lake basin. 
de los grupos de cazadores-recolectores de la región durante el Holoceno Tardío y su vinculación con las fluctuaciones ambientales experimentadas en Patagonia durante dicho periodo (Goñi 1991, 2000, 2010; Goñi y Barrientos 2004; Goñi et al. 2000-2002). En este sentido, Goñi et al. han postulado un modelo de poblamiento regional que sostiene que durante el Holoceno Tardío, particularmente durante los últimos 2500 años AP, se registra una tendencia general (aunque fluctuante) hacia la humedad ambiental decreciente, con condiciones de mayor aridez y circunscripción del agua a lugares puntuales. Dichas condiciones alcanzaron un punto máximo hacia ca. 900 años AP (sensu Stine 1994) durante el fenómeno climático global conocido como la Anomalía Climática Medieval (ACM). Este escenario habría dado lugar a cambios en los sistemas organizativos de los grupos de cazadores-recolectores locales, orientados a la reducción de la movilidad residencial, el constreñimiento espacial, el nucleamiento de poblaciones, y al mismo tiempo, una ampliación de los rangos de acción en materia de movimientos logísticos (Goñi 2000, 2010; Goñi y Barrientos 2004; Goñi et al. 2000-2002).

En este marco, este trabajo tiene como objetivos específicos evaluar y caracterizar la presencia de osteoartrosis, y artropatías afines (p.ej., nódulos de Schmorl) en las articulaciones mayores del esqueleto (columna vertebral, hombro, pelvis y rodilla) de individuos adultos, su distribución por sexo y edad y la identificación de cambios en las mismas a lo largo del tiempo (pre y post ACM). Estos objetivos se insertan en una investigación general cuyo fin último es indagar, en primer lugar, sobre los niveles de actividad física y estrés mecánico a los que estas poblaciones habrían estado sujetas y, en segundo lugar, evaluar si los cambios propuestos en las estrategias de movilidad y actividades (Goñi et al. 2000-2002) habrían provocado modificaciones en los patrones de uso del cuerpo, manifiestos de manera diferencial a nivel óseo.

En relación a lo dicho previamente, y partiendo de la información etnográfica general, la cual señala la existencia de la división sexual y etaria de las actividades en el interior de los grupos de cazadoresrecolectores (Binford 2001; Hooper et al. 2015), se plantea que en el área del Lago Salitroso, durante el Holoceno Tardío, tal división por sexo y edad habría existido, y se correspondería con diferencias en el uso del cuerpo de estos individuos según su sexo y edad, las cuales se manifestarán a nivel óseo. En relación con los cambios en la movilidad residencial y nucleamiento de poblaciones propuestos para momentos posteriores a la ACM, se postula que no se habrían producido cambios en la intensidad de las actividades realizadas por cada uno de los grupos considerados (sexo y edad de los individuos) a lo largo del Holoceno Tardío. Por lo tanto, no se esperan importantes diferencias en las frecuencias y severidad de las manifestaciones óseas que reflejan niveles de actividad dentro de cada grupo considerado entre periodos cronológicos. Sin embargo, dichos cambios habrían aparejado la ejecución de nuevas actividades tales como el uso de mano y molinos, y la producción cerámica. Esto habría afectado de manera diferencial a determinados grupos de sexo y edad, lo que se plasmaría a nivel del esqueleto en manifestaciones óseas de distinto tipo, y en una distribución diferencial de las articulaciones afectadas.

\section{Investigaciones Arqueológicas en el Lago Salitroso}

Los resultados de más de dos décadas de investigaciones arqueológicas en el noroeste de Santa Cruz, sustentan la idea de que la cuenca del Lago Salitroso habría funcionado como un espacio de uso eminentemente residencial, con asentamientos recurrentes y relativamente permanentes, en especial durante el último milenio del Holoceno Tardío (ca. 1200-900 AP sensu Stine 1994) (Cassiodoro 2011; García Guraieb 2010; Goñi 2010). El registro arqueológico de la cuenca presenta numerosos sitios a cielo abierto, algunos de ellos emplazados en médanos edafizados con molles (Schinus sp.) y a arbustos altos utilizados como reparo y leña (Goñi 2010). En ellos se ha identificado una amplia variedad de artefactos líticos, que incluyen desechos de talla, núcleos e instrumentos como raspadores, puntas de proyectil, raederas y cuchillos confeccionados en materias primas locales y de fuentes distantes; así como la presencia de artefactos poco transportables tales como manos y molinos, y artefactos cerámicos de manufactura local, interpretados como equipamiento del espacio (Cassiodoro et al. 2004; Goñi 2010; Goñi et al. 20002002). A su vez, se han recuperado restos faunísticos, con predominancia de guanaco, y en menor frecuencia de otras especies tales como choique, roedores, entre otros (Cassiodoro et al. 2004). La frecuencia relativamente alta de este tipo de sitios en la cuenca del Lago Salitroso, con cronologías que los ubican entre ca. 1500 y 500 años AP, brinda sustento a la hipótesis planteada de un uso predominantemente residencial, en el que se habrían concentrado las poblaciones humanas durante el Holoceno Tardío final (Cassiodoro 2011; Cassiodoro et al. 2004; Goñi 2010).

Al registro previamente descrito, se suma la abundancia y concentración de estructuras de entierro, ya sean acumulaciones de piedra a cielo abierto o incluidas en pequeños reparos naturales de piedra (García Guraieb 2010). El registro mortuorio brinda información clave para analizar a la cuenca como un lugar con especial interés residencial permanente 
o semi-permanente. Hasta el momento se han identificado 37 estructuras funerarias representadas por tres modalidades de entierro ${ }^{2}$, que pueden englobarse en tres momentos cronológicos diferentes: un grupo más temprano representado por entierros en "nichos" y entierros bajo bloque -EBB- (ca. 2600 - 2200 años AP, $n=10$ estructuras); un grupo minoritario de chenques iniciales (ca. $1500-1200$ años AP, $n=2$ chenques), y uno mayoritario de chenques tardíos (ca. 800 - 350 años AP, $n=25$ chenques), cuya cronología los sitúa con posterioridad a la ACM (García Guraieb 2010; García Guraieb et al. 2015; Goñi et al. 20002002). Las estructuras más tempranas suelen ser entierros individuales, mientras que los chenques tardíos se presentan principalmente como entierros múltiples y con evidencias de reutilización a lo largo de varios siglos (García Guraieb et al. 2018; Goñi et al. 2000-2002). Actualmente, la colección osteológica recuperada cuenta con aproximadamente 97 individuos de ambos sexos y de todas las edades, 71 de los cuales corresponden al grupo de chenques tardíos (García Guraieb et al. 2015). En cuanto a su distribución sexo-etaria, se destaca que en el grupo de estructuras de entierro más temprano (ca. 2600-2200 años AP), nichos y EBB, se encuentran mayormente representados los individuos adultos de ambos sexos, con predominancia de los individuos masculinos, y se observa la ausencia de individuos subadultos menores a 15 años (García Guraieb 2010; García Guraieb et al. 2015). Contrariamente, los chenques tardíos (ca. 800 -350 años AP) reflejan una distribución de sexos más pareja, con un leve predominio de individuos femeninos (García Guraieb et al. 2015) y una alta representación de individuos subadultos menores a 20 años, con una elevada cantidad de individuos menores a 10 años (Bernal et al. 2004; García Guraieb 2010; Guichón Fernández 2016). Este cambio en la composición sexoetaria de los chenques tardíos, sumado al hecho de que contienen mayoritariamente inhumaciones primarias, múltiples y extendidas, son las características del registro mortuorio que más fuertemente sustentan la idea de un uso residencial de la cuenca y la mencionada reducción de la movilidad en tiempos post ACM (García Guraieb et al. 2015, 2018; Goñi 2000, 2010; Goñi y Barrientos 2004; Goñi et al. 2000-2002).

El registro bioarqueológico del Lago Salitroso ha sido analizado empleando distintas líneas de evidencia, entre las que se destacan los estudios de prácticas mortuorias (Cassiodoro y García Guraieb 2009; García Guraieb et al. 2018), los análisis paleodemográficos y paleopatológicos (Bernal et al. 2004; García Guraieb 2006, 2010; García Guraieb y Maldonado 2014; García Guraieb et al. 2015, 2018; Goñi et al. 2003-2005), los paleodietarios (Tessone et al. 2015) y recientemente, los paleogenéticos (Arencibia et al. 2019). Dichos estudios, permitieron sostener que la población asentada en la cuenca estuvo sujeta a una diversidad de condiciones patológicas y traumáticas, aunque en bajas frecuencias, resultado de los cambios en la estrategia de movilidad y en la modalidad de ocupación de la cuenca durante el último mileno (García Guraieb 2010; García Guraieb y Maldonado 2014; Goñi et al. 2003-2005). No obstante, hasta el momento no se han realizado estudios de artropatías en la colección del Lago Salitroso que contribuyan a evaluar las implicancias de tales cambios en la movilidad y uso del espacio, desde la perspectiva del uso del cuerpo, el estrés mecánico y los patrones de actividad.

\section{Materiales y Métodos}

\section{Muestra}

A los fines de seleccionar los individuos que integran la muestra, se emplearon las asignaciones de sexo y edad realizadas en estudios previos, para las cuales se utilizaron criterios óseos y dentales de uso habitual en Bioarqueología (ver detalles de métodos y resultados en García Guraieb 2010; García Guraieb et al. 2007, 2015). En esta primera instancia se incluyeron a los individuos que cumpliesen el criterio de Waldron (2009) que establece condiciones de integridad anatómica de al menos el $60 \%$ del esqueleto. En la Tabla 1 se detallan los 14 individuos adultos (mayores a 20 años), de ambos sexos (seis femeninos y ocho masculinos), cuya cronología abarca el rango temporal desde los ca. 2600 a los 350 años AP. Si bien se trata de una muestra pequeña, ya que el objetivo fue realizar una primera aproximación al estudio de esta patología en la colección, se optó por dividirla en criterios amplios. De esta forma, siguiendo los criterios de estratificación de la muestra para el análisis de OA delineados por Weiss y Jurmain (2007), los individuos fueron discriminados por sexo, por edad y por cronología. En términos etarios, los individuos fueron divididos en dos grupos: menores a 40 años y mayores a 40 años. La división en estos dos grupos etarios responde, por un lado, a que justamente por tratarse de una muestra pequeña no permite realizar subdivisiones de edad más acotadas, y por el otro, a que la bibliografía epidemiológica reconoce el umbral de manifestación de la OA a partir de los 40 años aproximadamente (Carmona Ortells 2010). En términos cronológicos, la división responde a que la composición sexo-etaria de los entierros varía con el tiempo, y por lo tanto consideramos debía ser tenido en cuenta a los fines de controlar en lo posible dicho factor. Por tal motivo, los individuos fueron agrupados en dos bloques temporales: pre ACM (ca. 2600-1200 años AP) y post ACM (ca. 800-350 años AP).

Para esta primera etapa del análisis se incluyeron las articulaciones correspondientes a la columna vertebral, y a las articulaciones apendiculares del hombro (incluida 
Tabla 1. Composición de la muestra discriminada por individuo, modalidad de entierro, grupo cronológico, sexo y edad.

Sample composition by individual, type of burial, chronological group, sex, and age.

\begin{tabular}{ccccccc}
\hline Individuo & $\begin{array}{c}\text { Modalidad } \\
\text { Entierro }\end{array}$ & $\begin{array}{c}\text { Grupo } \\
\text { Cronológico } \\
\text { años AP }\end{array}$ & Bloque temporal & Sexo & $\begin{array}{c}\text { Rango de edad } \\
\text { (en años) }\end{array}$ & $\begin{array}{c}\text { Grupo } \\
\text { Etario }\end{array}$ \\
\hline SAC 4-N1-1 & Nicho & $2600-2200$ & Pre ACM & F & $24-30$ & $<40$ \\
\hline SAC 4-N3-1 & Nicho & $2600-2200$ & Pre ACM & M & $50+$ & $>40$ \\
\hline SAC 4-N4-1 & Nicho & $2600-2200$ & Pre ACM & M & $50+$ & $>40$ \\
\hline SAC 1-11-1 & EBB & $2600-2200$ & Pre ACM & M & $50+$ & $>40$ \\
\hline SAC 4-2-1 & EBB & $2600-2200$ & Pre ACM & F & $45-49$ & $>40$ \\
\hline SAC 1-7-1 & CH I & $1500-1200$ & Pre ACM & M & $40+$ & $>40$ \\
\hline SAC 1-7-2 & CH I & $1500-1200$ & Pre ACM & M & $20-25$ & $<40$ \\
\hline SAC 1-1-3 & CH T & $800-350$ & Post ACM & F & $35-39$ & $<40$ \\
\hline SAC 1-1-B & CH T & $800-350$ & Post ACM & F & $40-44$ & $>40$ \\
\hline SAC 2-8-4 & CH T & $800-350$ & Post ACM & M & Adulto & $>40$ \\
\hline SAC 4-1-1 & CH T & $800-350$ & Post ACM & F & $40-44$ & $>40$ \\
\hline SAC 8-3-4 & CH T & $800-350$ & Post ACM & M & $25-35$ & $<40$ \\
\hline SAC 10-4-1 & CH T & $800-350$ & Post ACM & M & $25-30$ & $<40$ \\
\hline SAC 30-1-1 & CH T & $800-350$ & Post ACM & F & $18-24$ & $<40$ \\
\hline
\end{tabular}

CHT: Chenque tardío; CH I: Chenque inicial; EBB: Entierro Bajo Bloque; F: Femenino; M: Masculino. El nombre de cada individuo contiene la nomenclatura del sitio y entierro al que pertenece. Así, el individuo SAC 8-3-4 es el individuo 4 del chenque 3 del sitio 8 .

la articulación acromioclavicular), la pelvis y la rodilla. En el caso de las articulaciones apendiculares la elección se basó en tres criterios: que se trata de las articulaciones sinoviales de mayor tamaño del esqueleto (Bridges 1993), que representan diferentes sectores del mismo (i.e., miembros superiores e inferiores) y que son las que mejor cumplían el criterio de integridad anatómica de Waldron (2009).

Los elementos óseos analizados representan un total de 12 sacros, 79 vértebras cervicales, 147 torácicas y 63 lumbares (1.598 superficies articulares vertebrales, incluida la articulación del sacro con la quinta vértebra lumbar), 27 articulaciones del hombro (incluyen 79 regiones articulares: 25 cabezas de húmero, 23 cavidades glenoideas, 17 acromiones y 14 extremidades acromiales de la clavícula), 27 articulaciones de pelvis (incluyen 47 regiones articulares: 23 cabezas de fémur y 24 acetábulos del coxal) y 22 articulaciones de rodilla (incluyen 56 regiones articulares: 20 epífisis distales del fémur, 14 facetas articulares de la rótula y 22 epífisis proximales de la tibia).

\section{Métodos}

La identificación de la OA se realizó a partir del análisis macroscópico de las lesiones proliferativas y las alteraciones inflamatorias presentes en las superficies articulares de la columna vertebral y de las articulaciones apendiculares (las superficies articulares no observables fueron contabilizadas, pero no incluidas en el análisis del presente trabajo). Cabe destacar que, a pesar de que las modificaciones óseas en las articulaciones cartilaginosas, tal como son las articulaciones intervertebrales del cuerpo vertebral, no son osteoartrósicas por estricta definición (Jurmain 1990; Waldron 2009), su etiología y patogénesis son similares a la osteoartrosis (Burt et al. 2013; Jurmain 1990; Waldron 2009). Por tal motivo, y dado que el uso del término osteoartrosis es aceptado por diversos investigadores para describir las lesiones en los cuerpos vertebrales (Bridges 1991, 1994; Jurmain 1990; Larsen 1995, 1997; Lieverse et al. 2016; Merbs 1983; Ortner 2003), se lo utilizó en el presente trabajo en este sentido más amplio.

En el caso de las vértebras, se consideraron separadamente las superficies articulares de las carillas (superior derecha, superior izquierda, inferior derecha e inferior izquierda) del cuerpo vertebral (superior e inferior) (Resnick 2002; Rojas-Sepúlveda et al. 2008). Se tuvo en cuenta el sacro para las superficies de articulación con la quinta vértebra lumbar. Para los huesos largos se observaron de manera separada la epífisis distal de la epífisis proximal y en los huesos planos (pelvis y escápula) se tomaron en cuenta las regiones articulares correspondientes al acetábulo en coxales, a la cavidad glenoidea y acromion en escápula y a la extremidad acromial de la clavícula. A su vez, se tuvo en cuenta la bilateralidad en los miembros superiores e inferiores para dar cuenta de la potencial 
diferencia entre los lados. Asimismo, los miembros superiores fueron analizados separadamente de los miembros inferiores, dado que cada uno de ellos está relacionado con la ejecución de actividades diferentes, resultado de distintas demandas mecánicas (Bridges 1991; Scabuzzo 2010; Weiss y Jurmain 2007).

De esta forma, se relevaron por separado las lesiones proliferativas -osteofitos, remodelación del margen de la carilla articular del arco vertebral, labiado o lipping y eburnación- (Arrieta y Mendonça 2011; Rogers y Waldron 1995; Rojas-Sepúlveda et al. 2008; Sofaer Derevenski 2000) de las alteraciones inflamatorias -porosidad o pitting-, ya que dan cuenta de procesos de deterioro articular diferentes (Luna et al. 2017; Sofaer Derevenski 2000). Se utilizó, para cada lesión proliferativa en particular, una escala de cuatro grados de severidad: $0=$ ausente; $1=$ leve; $2=$ moderado $y$ $3=$ severo (Arrieta y Mendonça 2011; Rojas-Sepúlveda et al. 2008; Sofaer Derevenski 2000). Las escalas utilizadas para cada rasgo dan cuenta de la extensión de las lesiones en la superficie articular y fueron seleccionadas dado que son las comúnmente utilizadas en los análisis realizados por otros investigadores, lo cual permitirá realizar comparaciones de resultados. La porosidad no se consideró una variable determinante para el diagnóstico positivo de la OA, dada la dificultad para diferenciarla de los deterioros tafonómicos (RojasSepúlveda et al. 2008; Sofaer Derevenski 2000). De todas formas, se relevó la presencia/ausencia y severidad de la porosidad a partir de una escala de cinco grados: $0=\sin$ porosidad; $1=$ agujeros $<0,5 \mathrm{~mm}$ de diámetro; $2=$ agujeros $0,5-1 \mathrm{~mm}$ de diámetro; $3=$ agujeros $>1$ $\mathrm{mm}$ y $\leq 1,5 \mathrm{~mm}$ de diámetro y $4=$ agujeros $>1,5 \mathrm{~mm}$ de diámetro (Sofaer Derevenski 2000). Se utilizó este sistema de registro dado que utiliza medidas cuantitativas, las cuales facilitan la evaluación de la extensión de la porosidad y reduce su confusión con los efectos generados por procesos postdepositacionales.

Se consideró a la eburnación como patognomónica de la patología y un marcador de la severidad de la misma (Rogers y Waldron 1995; Waldron 2009). Asimismo, siguiendo a Rogers y Waldron (1995), la presencia en una superficie articular de al menos dos de las lesiones proliferativas mencionadas también fue considerada indicativa de OA. Las articulaciones apendiculares, al estar integradas por múltiples regiones articulares, ante la presencia de OA en una de dichas regiones articulares fue considerada suficiente para diagnosticar con OA a la articulación en general. Por último, los casos de OA fueron clasificados de acuerdo al grado de severidad. Para ello, siguiendo a Neves (1984), Rodrigues Carvalho (2004) y Scabuzzo (2010), se utilizó un sistema de clasificación de cuatro grados: $0=$ ausente; $1=$ leve (definición de márgenes articulares con afilamiento de los bordes, acompañado o no de crecimiento óseo incipiente, menor a $1 \mathrm{~mm}$ ); 2 =moderado (los márgenes de la articulación presentan labiado o lipping mayores a $1 \mathrm{~mm}$ y se denota desgaste en la superficie articular); y $3=$ severo (las proyecciones óseas son más acentuadas, mayores a los $2 \mathrm{~mm}$, a la vez que es mayor el desgaste de la superficie articular. Puede observarse la presencia de eburnación). Al asignar un grado de severidad de la OA a la articulación en su conjunto, en el caso de las articulaciones apendiculares, cuyas regiones articulares pueden exhibir grados diferentes de severidad, se tomó el valor del grado de severidad más elevado.

A efectos de evaluar el error intraobservador, se realizaron dos series de observaciones por parte del mismo operador (M.M.) con un lapso de dos meses entre cada una. El error se calculó a través de la estimación del porcentaje de acuerdo entre las observaciones, la obtención del Índice Kappa de Cohen y su interpretación empleando la escala de Landis y Koch (1977). Cuando se observaron discordancias entre sesiones, los elementos en cuestión fueron nuevamente analizados para obtener una evaluación definitiva (Arrieta y Mendonça 2011). Los datos obtenidos para el cálculo del error intraobservador fueron procesados a través del programa estadístico SPSS Statistics 22.

A partir de los datos relevados, se realizó el cálculo de las frecuencias generales, a nivel de los individuos (FI) y de las superficies articulares de las carillas y cuerpos vertebrales y de las regiones articulares de las articulaciones apendiculares (FV: frecuencias vertebrales y FA: frecuencias articulaciones apendiculares) de los casos positivos de OA. Dichas cuantificaciones fueron realizadas por sexo, grupo etario ( $<40$ años y $>40$ años), bloque temporal (pre ACM y post ACM) y lateralidad (para el caso de las articulaciones apendiculares). $\mathrm{La}$ significación de las diferencias existentes en la expresión de la OA entre los grupos de edad, sexo, bloque temporal y lateralidad se calculó aplicando el Test de Chi-cuadrado $\left(\mathrm{X}^{2}\right)$ o la Prueba Exacta de Fisher (según correspondiera), estableciéndose un nivel de significación de $\mathrm{p} \leq 0,05$. Los datos obtenidos fueron procesados a través del programa PAST 3.20.

\section{Resultados}

\section{Evaluación del error intraobservador}

El cálculo del error intraobservador brindó como resultado un índice de acuerdo por encima del 0,61 para K (Cohen), por lo tanto, dentro de los rangos de acuerdo "sustancial"y "muy bueno" según la escala de Landis y Koch (1977). Como el error intraobservador obtenido fue pequeño, se utilizó la segunda serie de observaciones, dado que se entiende expresa una mayor experiencia por parte del observador. 


\section{Osteoartrosis en la columna vertebral}

En la Tabla 2 se exhiben los resultados de la frecuencia de OA por sexo, grupo de edad, bloque temporal y región de la columna vertebral de los 14 individuos analizados. El 85,7\% de ellos (12/14) fueron diagnosticados positivamente con OA en la columna vertebral. A su vez, fueron analizadas 289 vértebras y 1598 superficies articulares, de las cuales 85 vértebras $(29,4 \%)$ y 211 superficies articulares $(13,2 \%)$ fueron diagnosticadas con OA (Figura 2). Las superficies articulares afectadas corresponden a 168 carillas articulares $(168 / 1092,15,4 \%)$ y a 43 cuerpos vertebrales $(43 / 506,8,5 \%)$. La región lumbar exhibe la mayor frecuencia de OA (vértebras: $33 / 63,52,4 \%$; carillas articulares: 85/250, 34,0\%; cuerpos vertebrales: 21/128, $16,4 \%$ ), seguido por la región torácica, particularmente el segmento inferior (vértebras: $36 / 147,24,5 \%$; carillas articulares: 72/540, 13,3\%; cuerpos vertebrales: $12 / 261$, 4,6\%) (Tabla 2).
En relación a la severidad de la OA, las carillas articulares exhiben, en su mayoría, severidad leve (124/1092, 11,4\%), con bajas frecuencias de afectación moderada (34/1092, 3,1\%) y severa (10/1092, $0,9 \%)$. De la misma forma, si bien casi la totalidad de los cuerpos vertebrales no presentan $\mathrm{OA}$ (grado 0 ), aquellos diagnosticados positivamente exhiben severidad leve $(32 / 506,6,3 \%)$ y moderada $(11 / 506,2,2 \%)$ (Figuras 3 y 4). Cabe destacar que las superficies articulares que exhiben mayores grados de severidad, ya sean carillas articulares o cuerpos vertebrales, se ubican en las regiones de la columna con mayores frecuencias de $\mathrm{OA}$, es decir, el segmento inferior de la región torácica y la región lumbar.

En cuanto a la relación entre el sexo de los individuos y la presencia de OA, se observa que los individuos de sexo masculino exhiben una mayor frecuencia de $\mathrm{OA}$ en las superficies articulares vertebrales (128/944, 13,6\%) que los individuos femeninos $(83 / 654,12,7 \%)$ (Tabla 2). Sin embargo, el análisis de la frecuencia de OA entre

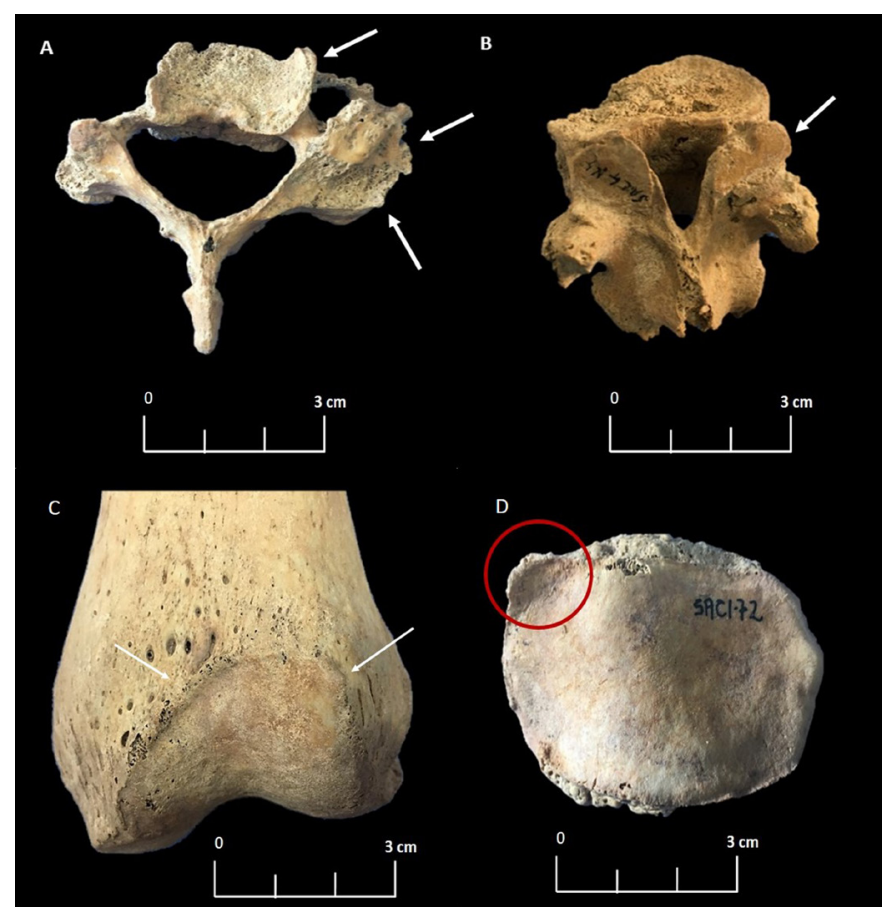

Figura 2. Casos de OA en la muestra analizada. (A) Vértebra C4 (SAC 4-N3-1, Adulto Mayor. Masculino). Carilla superior derecha con OA (eburnación, porosidad y remodelación del margen). (B) Vértebra T12 (SAC 4-N4-1, Adulto Mayor. Masculino). Carilla superior derecha con OA (eburnación). (C) Epífisis distal fémur izquierdo (SAC 4-1-1, Adulto Joven Femenino) con OA (eburnación en el margen superior derecho (vista frontal) y lipping. (D) Faceta articular-rótula izquierda (SAC 1-7-2, Adulto Joven Masculino) con OA (osteofitos marginales y lipping).

OA cases in the sample analyzed. (A) Vertebra C4 (SAC4-N3-1, Older Adult. Male). Upper right apophyseal facet with $O A$ (eburnation, porosity and facet remodeling). (B) Vertebra T12 (SAC 4-N4-1, Older Adult Male). Right upper apophyseal facet with OA (eburnation). (C) Distal epiphysis of left femur (SAC 4-1-1, Young Adult Female) with OA (eburnation in the upper right margin (frontal view) and lipping. (D) Articular facet-left patella (SAC 1-7-2, Young Adult Male) with OA (marginal osteophytes and lipping). 


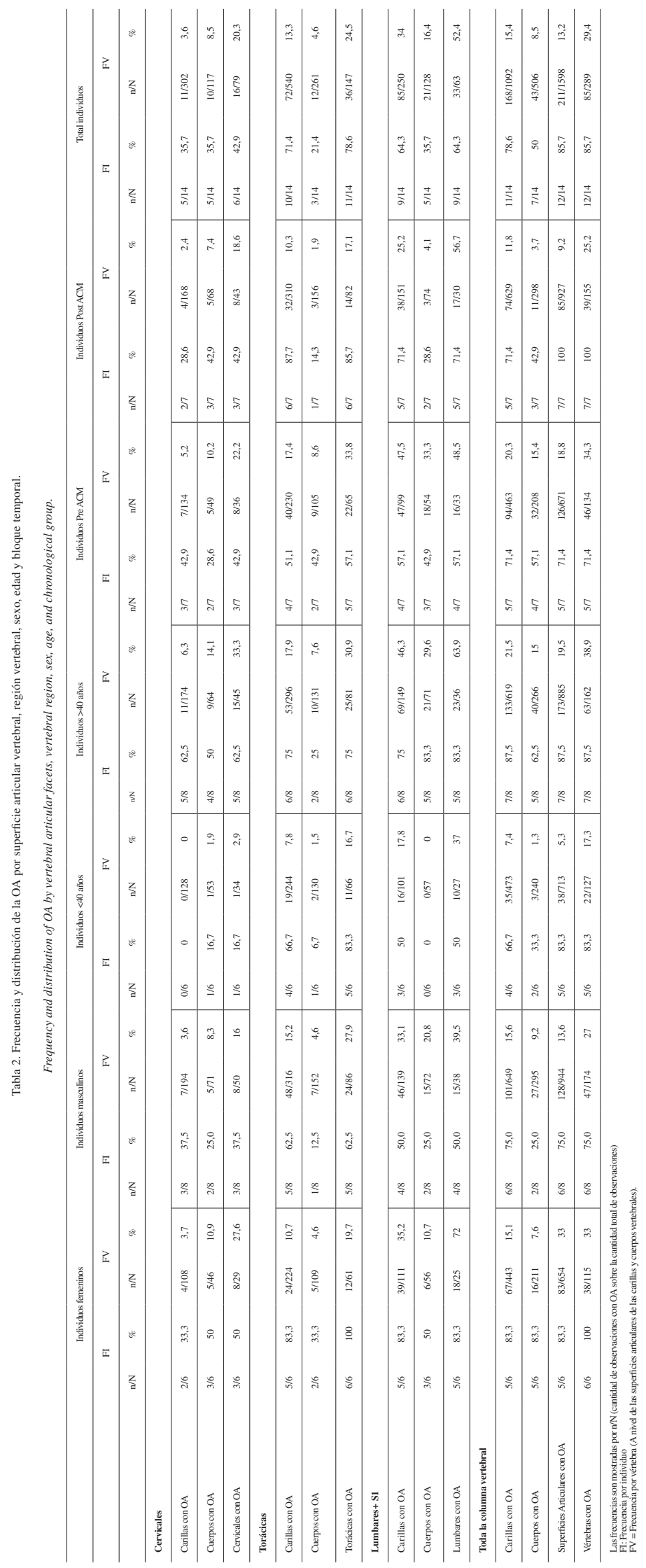


Cantidad de carillas articulares con OA distribuídas por vértebra y grado severidad

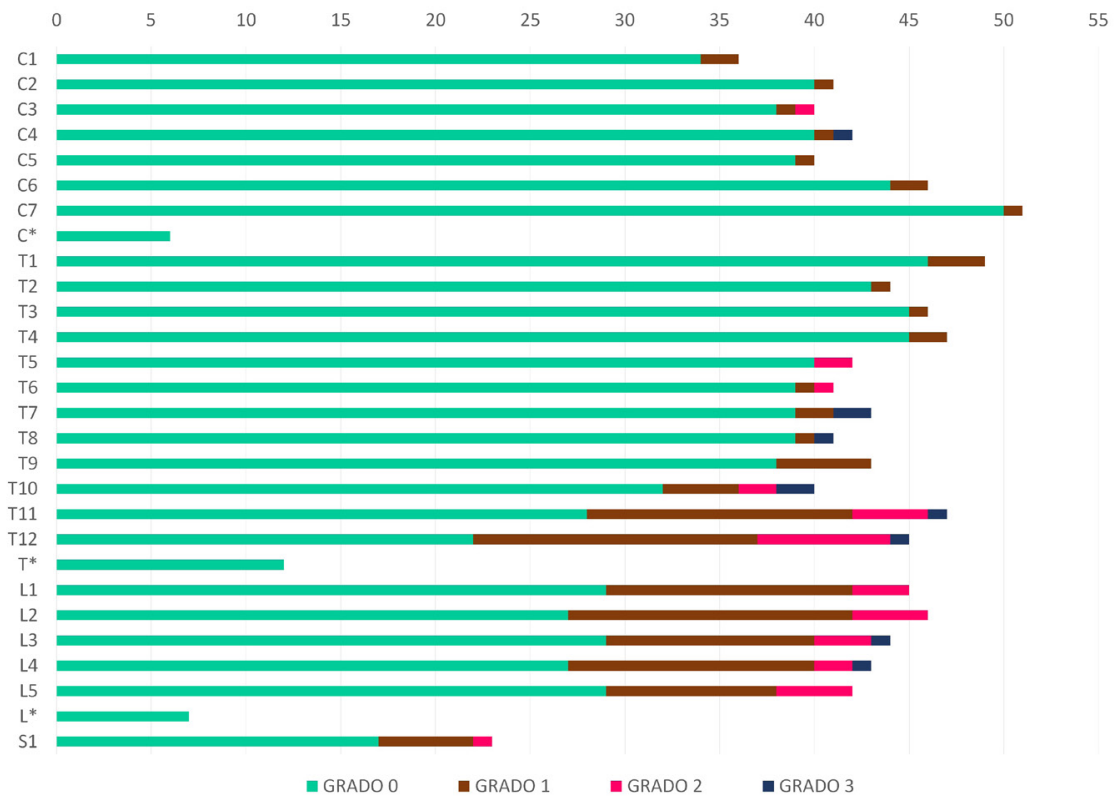

Figura 3. Cantidad de carillas articulares vertebrales diagnosticadas con OA distribuidas por vértebra y grado de severidad en el conjunto analizado. $C^{*}, T^{*}$ y L* representan las vértebras no identificadas en cada región de la columna vertebral.

Number of vertebral articular facets diagnosed with $O A$ distributed by vertebra and degree of severity in the set analyzed. $C$ *, $T *$ and $L *$ represent the unidentified vertebrae in each region of the spine.

Cantidad de cuerpos vertebrales con OA distribuídos por vértebra y

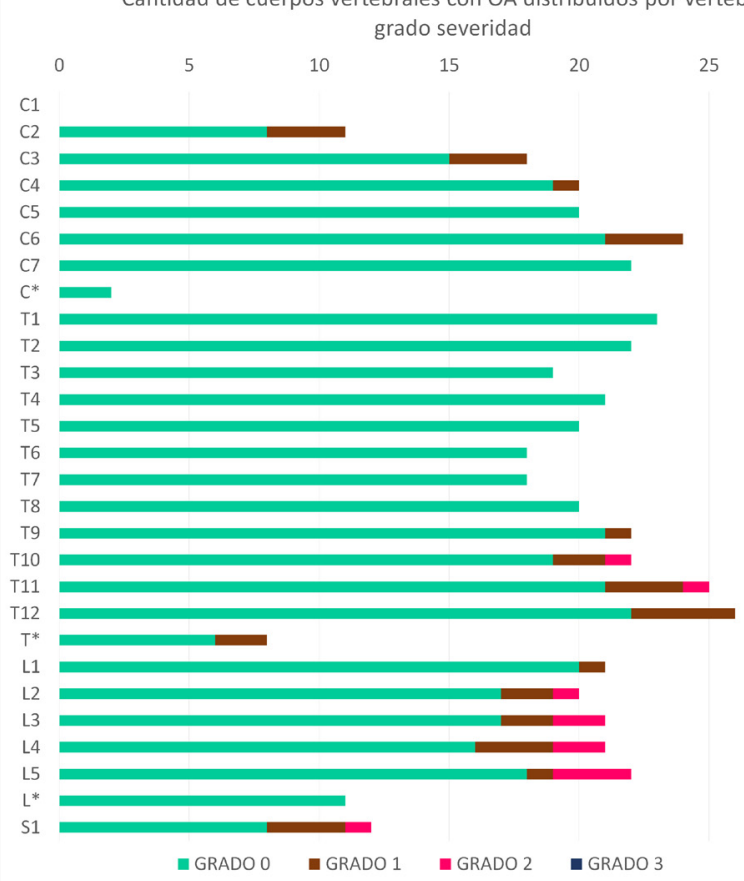

Figura 4. Cantidad de cuerpos vertebrales diagnosticados con OA distribuidos por vértebra y grado de severidad en el conjunto analizado. $\mathrm{C}^{*}, \mathrm{~T}^{*}$ y $\mathrm{L}^{*}$ representan las vértebras no identificadas en cada región de la columna vertebral.

Number of vertebral bodies diagnosed with $O A$ distributed by vertebra and degree of severity in the set analyzed. $C^{*}, T^{*}$ and $L^{*}$ represent the unidentified vertebrae in each region of the spine. 
sexos no arrojó diferencias estadísticamente significativas a nivel de las carillas $\left(\mathrm{X}^{2}=1,01 ; \mathrm{gl}=1 ; \mathrm{p}=0,34\right)$, ni a nivel de los cuerpos vertebrales $\left(X^{2}=1,05 ; g l=1 ; p=0,31\right)$ (Tabla 3).

Tabla 3. Valores de p obtenidos en las Prueba de Chi-cuadrado/ Prueba Exacta de Fisher para las manifestaciones en las superficies articulares de las tres regiones de la columna vertebral de los individuos de acuerdo al sexo, el grupo etario y el bloque cronológico.

P-values from Chi-square/ Fisher's Exact Test for manifestations on the articular surfaces of the three regions of the spine of individuals according to sex, age group, and chronological group.

\begin{tabular}{|c|c|c|c|c|}
\hline & & Masculino & $>40$ & Post ACM \\
\hline \multirow{3}{*}{ Carillas Cervicales } & Femenino & 0,54 & - & - \\
\hline & $<40$ & - & $0,02 *$ & - \\
\hline & Pre ACM & - & - & 0,19 \\
\hline \multirow{3}{*}{ Cuerpos Cervicales } & Femenino & 0,65 & - & - \\
\hline & $<40$ & - & $0,002 *$ & - \\
\hline & Pre ACM & - & - & 0,58 \\
\hline \multirow{3}{*}{ Carillas Torácicas } & Femenino & 0,06 & - & - \\
\hline & $<40$ & - & 3,7 & - \\
\hline & Pre ACM & - & - & $0,02 *$ \\
\hline \multirow{3}{*}{ Cuerpos Torácicos } & Femenino & 0,86 & - & - \\
\hline & $<40$ & - & $0,002 *$ & - \\
\hline & Pre ACM & - & - & $0,01 *$ \\
\hline \multirow{3}{*}{ Carillas Lumbares } & Femenino & 0,71 & - & - \\
\hline & $<40$ & - & 4,26 & - \\
\hline & Pre ACM & - & - & $0,002 *$ \\
\hline \multirow{3}{*}{ Cuerpos Lumbares } & Femenino & 0,06 & - & - \\
\hline & $<40$ & - & 5,05 & - \\
\hline & Pre ACM & - & - & 1,58 \\
\hline \multirow{3}{*}{ Total Carillas } & Femenino & 0,34 & - & - \\
\hline & $<40$ & - & $0,02 *$ & - \\
\hline & Pre ACM & - & - & $0,0001 *$ \\
\hline \multirow{3}{*}{ Total Cuerpos } & Femenino & 0,31 & - & - \\
\hline & $<40$ & - & $0,002 *$ & - \\
\hline & Pre ACM & - & - & 3,4 \\
\hline
\end{tabular}

$(*=\mathrm{p}<0,05)$

En lo que respecta a la edad, se observa que los individuos mayores a 40 años presentan mayores frecuencias de afectación $(173 / 885,19,5 \%)$ que los individuos menores de 40 años (38/713, 5,3\%) (Tabla 2). La Prueba Exacta de Fisher aplicada para las frecuencias de OA halladas en la columna vertebral de ambos grupos etarios arrojó diferencias estadísticamente significativas para las carillas vertebrales cervicales $(\mathrm{p}=0,02)$, para los cuerpos vertebrales cervicales $(\mathrm{p}=0,002)$ y para los cuerpos vertebrales torácicos $(\mathrm{p}=0,002)$ (Tabla 3$)$.
Por último, al considerar los bloques temporales, se observó que los individuos correspondientes a momentos previos a la ACM exhiben mayores frecuencias (126/671, $18,8 \%$ ) que aquellos de momentos posteriores a la $\mathrm{ACM}$ (85/927, 9,2\%) (Tabla 2). De acuerdo con los resultados de las pruebas de $\mathrm{X}^{2}$ y la Prueba Exacta de Fisher, las diferencias entre individuos de los bloques temporales fueron estadísticamente significativas en el caso de las carillas vertebrales torácicas $\left(X^{2}=5,71 ; g l=1 ; p=0,02\right)$, para los cuerpos vertebrales torácicos (Prueba Exacta de Fisher, $\mathrm{p}=0,01)$ y para las carillas vertebrales lumbares $\left(\mathrm{X}^{2}=13,26 ; \mathrm{gl}=1 ; \mathrm{p}=0,0002\right)$ (Tabla 3$)$.

\section{Osteoartrosis en las articulaciones apendiculares}

En la Tabla 4 se presentan los resultados de la frecuencia de OA por sexo, grupo de edad y bloque temporal correspondientes al total de las articulaciones apendiculares de los 14 individuos analizados, mientras que las Figuras 5 y 6 discriminan dichos datos por lateralidad y severidad en las articulaciones completas y en las regiones articulares de cada una de ellas, respectivamente.

En cuanto a las frecuencias totales (Tabla 4), el $71,4 \%$ (10/14) de los individuos fueron diagnosticados positivamente con OA en al menos una de las articulaciones aquí analizadas. Se relevaron 76 articulaciones apendiculares y 182 regiones articulares, de las cuales 20 articulaciones $(26,3 \%)$ y 28 regiones articulares $(15,4 \%)$ fueron diagnosticadas positivamente con OA. Las articulaciones con OA corresponden a siete articulaciones del hombro (7/27, 25,9\%), ocho a la pelvis $(8 / 27,29,6 \%)$ y cinco a la rodilla $(5 / 22,22,7 \%)$. En el hombro, las regiones articulares con OA corresponden tres a la cabeza de húmero $(3 / 25,12 \%)$, dos a la cavidad glenoidea $(2 / 23$, $8,7 \%)$, tres al acromion $(3 / 17,17,7 \%)$, y una a la extremidad acromial $(1 / 14,7,1 \%)$. En la pelvis, las regiones articulares que mostraron $O A$ se identificaron en tres cabezas de fémur $(3 / 23,13,0 \%)$ y en seis acetábulos del coxal $(6 / 24,25,0 \%)$. Por su parte, en la rodilla, las regiones articulares con OA fueron tres epífisis distales de fémur $(3 / 20,15,0 \%)$, cuatro facetas articulares de la rótula $(4 / 14,28,6 \%)$ y tres epífisis proximales de la tibia $(3 / 22,13,6 \%)$. De este modo, la pelvis es la articulación que exhibe la mayor frecuencia de $\mathrm{OA}$ a nivel de las articulaciones apendiculares y en términos de frecuencia relativa de regiones articulares afectadas $(9 / 47,19,1 \%)$ (Tabla 4$)$.

Si estas frecuencias se analizan discriminando por lateralidad y severidad, se observa a nivel de las articulaciones ciertas diferencias en las frecuencias de OA entre lateralidades, en las cuales el lado izquierdo exhibe mayor frecuencia de OA que el lado derecho $(15 / 41,36,59 \%$ y $6 / 37,16,22 \%$ respectivamente) (Figura 5). De igual forma, las regiones articulares del lado izquierdo exhiben mayores frecuencias de OA 


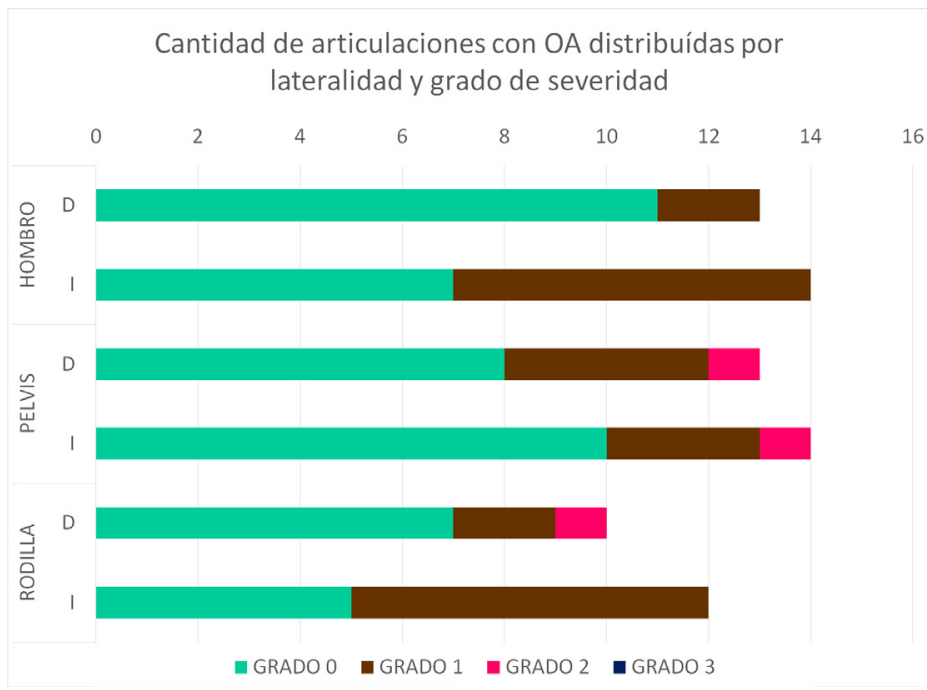

Figura 5. Cantidad de articulaciones apendiculares diagnosticadas con OA distribuidas por articulación, lateralidad y grado de severidad en el conjunto analizado.

Number of appendicular joints diagnosed with OA distributed by joint, laterality, and degree of severity in the set analyzed.

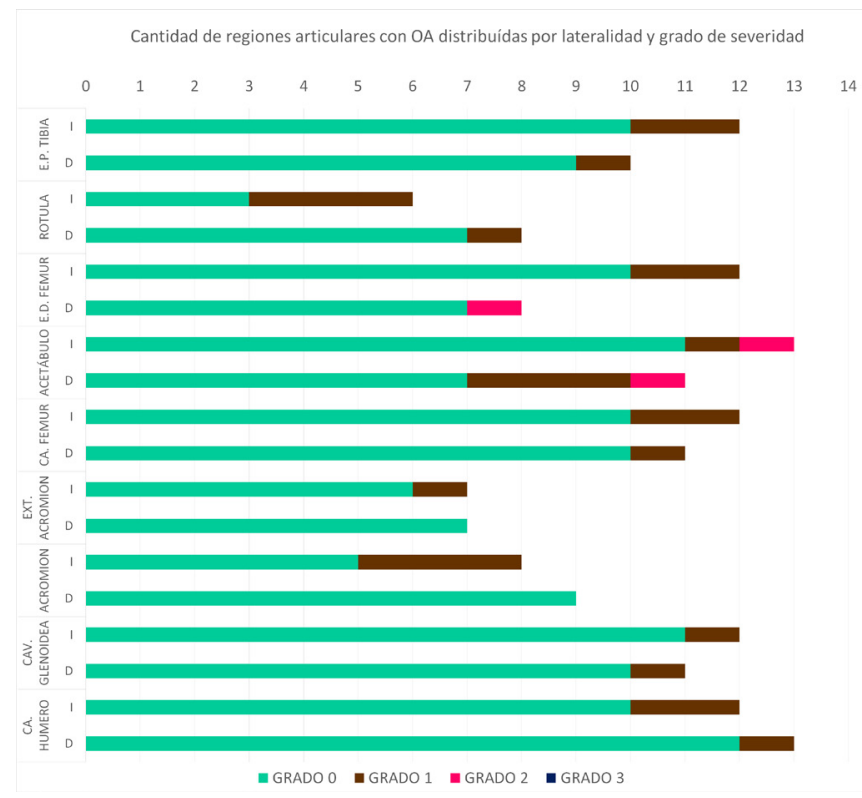

Figura 6. Cantidad de regiones articulares diagnosticadas con OA distribuidas por articulación, lateralidad y grado de severidad en el conjunto analizado.

Number of appendicular joint regions diagnosed with $O A$ distributed by joint, laterality, and degree of severity in the set analyzed. 


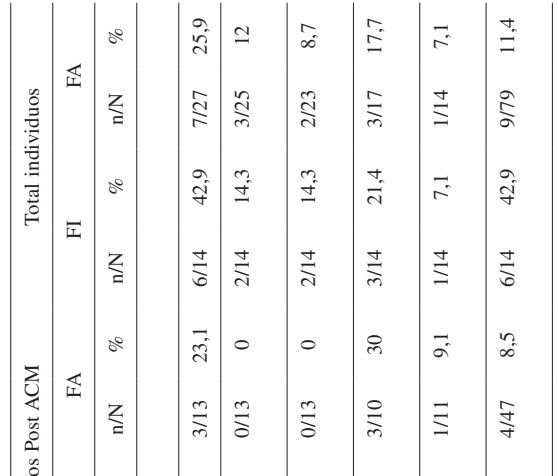
है:

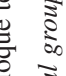
$\frac{0^{\circ}}{3}$

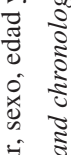
苛

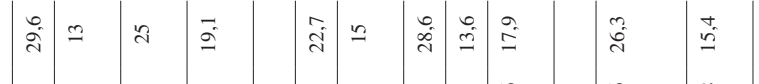

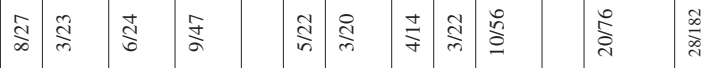

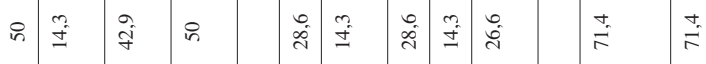

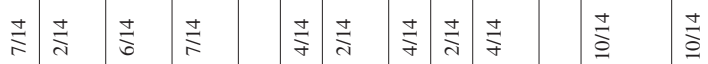

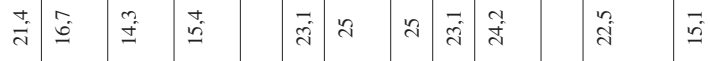

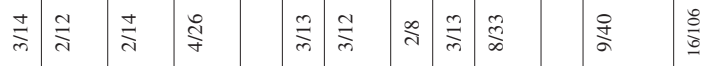

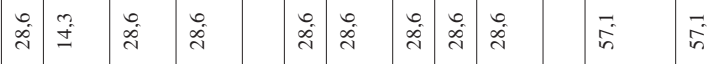

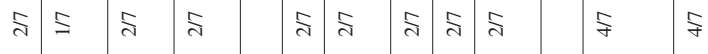

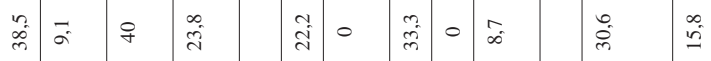

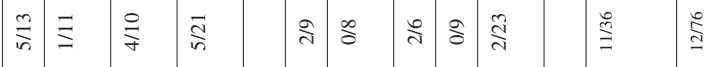

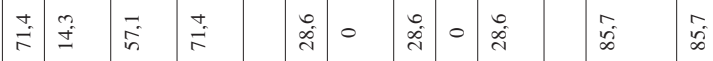
的年 $\frac{1}{5}$ 年

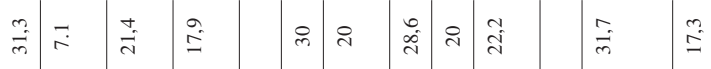

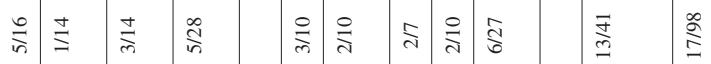

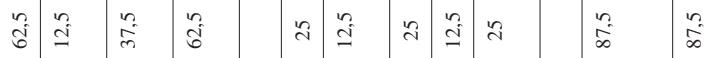

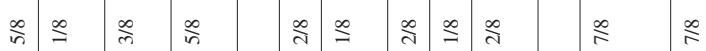

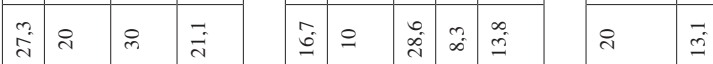

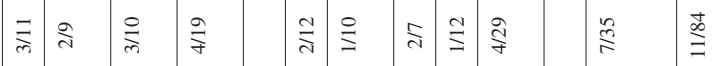

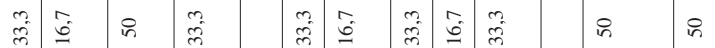

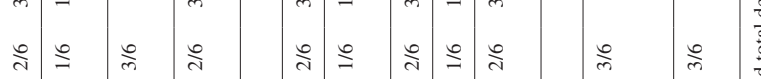

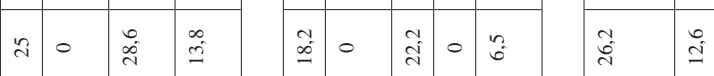

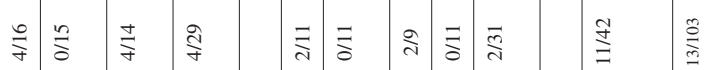
in 0 in in in

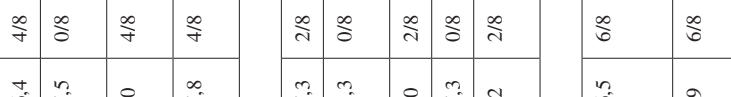

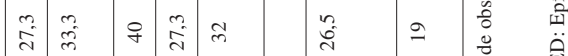

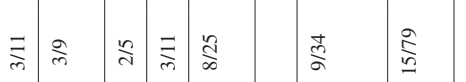

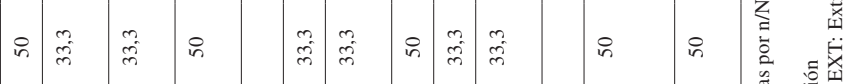

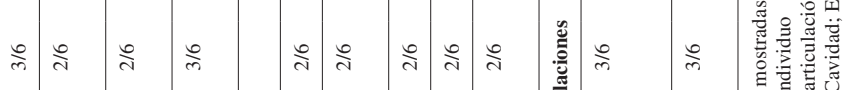

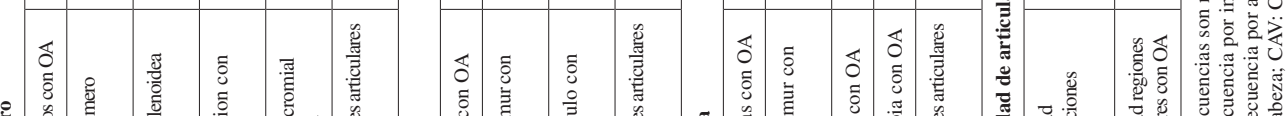

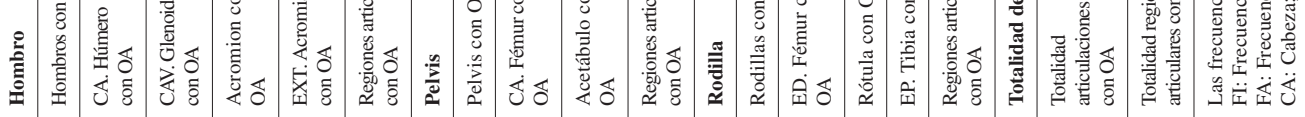


que el lado derecho (18/94, 19,15\% y 10/91, 10,99\% respectivamente) (Figura 6). Sin embargo, el test de $\mathrm{X}^{2}$ aplicado a las tres articulaciones apendiculares, así como a cada una de las regiones articulares, no arrojó diferencias estadísticamente significativas entre lateralidades $\left(X^{2}=3,28 ; \mathrm{gl}=1 ; \mathrm{p}=0,06\right.$ y $\mathrm{X}^{2}=2,11$; $\mathrm{gl}=1 ; \mathrm{p}=0,14$ respectivamente) (Tabla 5). Por su parte, al considerar la severidad de la OA, las articulaciones apendiculares de ambos lados exhiben, en su mayoría, severidad leve $(17 / 76,22,4 \%)$, con bajas frecuencias de severidad moderada $(3 / 76,3,9 \%)$ (Figura 5). De igual forma, las regiones articulares de las dos lateralidades exhiben severidad leve $(25 / 182,13,7 \%)$, hallándose OA de severidad moderada $(3 / 182,1,6 \%)$ en el acetábulo del coxal y en la epífisis distal del fémur (Figura 6).

La ausencia de diferencias estadísticas en la frecuencia de $\mathrm{OA}$ entre lateralidades en las articulaciones y regiones articulares apendiculares, permite combinar los lados, a los fines de analizar otras variables de la muestra como la edad, el sexo y los bloques temporales. Así, las frecuencias de OA en las articulaciones apendiculares son similares entre los individuos de ambos sexos, ya que en los individuos femeninos el 26,5\% (9/34) de las articulaciones presentan OA y en los individuos masculinos el 26,2\% (11/42) de las articulaciones tienen esta condición (Tabla 4). De acuerdo a las regiones articulares, los individuos femeninos exhiben mayor frecuencia de OA $(15 / 79,19,0 \%)$ que los individuos masculinos (13/103, 12,6\%) (Tabla 4). Sin embargo, el test de $\mathrm{X}^{2}$ aplicado tanto para las tres articulaciones apendiculares $\left(\mathrm{X}^{2}=0,0002 ; \mathrm{gl}=1 ; \mathrm{p}=0,99\right)$ como para las regiones articulares $\left(\mathrm{X}^{2}=1,19 ; \mathrm{gl}=1 ; \mathrm{p}=0,27\right)$ de los individuos de ambos sexos no arrojaron diferencias estadísticamente significativas (Tabla 5).

En lo que respecta a la edad, se observa que los individuos mayores a 40 años de edad presentan en las

Tabla 5. Valores de p obtenidos en las pruebas de Chi-cuadrado/Test de Fisher para las articulaciones apendiculares de los individuos de acuerdo al sexo, el grupo etario, el bloque cronológico y lateralidad.

$P$-values from Chi-square/ Fisher's Exact Test for manifestations in the appendicular joints of individuals according to sex, age group, chronological group, and laterality.

\begin{tabular}{lccccc}
\hline \multirow{5}{*}{ Masculino } & $>40$ & $\begin{array}{c}\text { Post } \\
\text { ACM }\end{array}$ & Izquierdo \\
\hline \multirow{2}{*}{ Articulaciones } & Femenino & 0,99 & - & - & - \\
\cline { 2 - 6 } & $<40$ & - & 0,2 & - & - \\
\cline { 2 - 6 } & Pre ACM & - & - & 0,25 & - \\
\cline { 2 - 6 } & Derecho & - & - & - & 0,06 \\
\hline \multirow{2}{*}{$\begin{array}{l}\text { Regiones } \\
\text { articulares }\end{array}$} & Femenino & 0,27 & - & - & - \\
\cline { 2 - 6 } & $<40$ & - & 0,2 & - & - \\
\cline { 2 - 6 } & Pre ACM & - & - & 0,9 & - \\
\cline { 2 - 6 } & Derecho & - & - & - & 0,14 \\
\hline
\end{tabular}

articulaciones apendiculares mayores frecuencias de afectación (13/41,31,7\%) que los individuos menores de 40 años $(7 / 35,20,0 \%)$ (Tabla 4). De igual forma, a nivel de las regiones articulares, los individuos mayores a 40 años presentan mayor frecuencia de OA $(17 / 98,17,3 \%)$ que los menores a 40 años $(11 / 84,13,1 \%)$ (Tabla 4$)$. Sin embargo, tampoco en este caso el test de $\mathrm{X}^{2}$ aplicado comparando los individuos de ambos grupos etarios arrojó diferencias estadísticamente significativas entre ellos, ni para las articulaciones $\left(X^{2}=1,62 ; \mathrm{gl}=1 ; \mathrm{p}=0,20\right)$ ni para las regiones articulares $\left(X^{2}=1,61 ; g l=1 ; p=0,20\right)$ (Tabla 5).

En cuanto a la comparación entre bloques temporales, los individuos correspondientes a momentos previos a la ACM exhiben mayor frecuencia de articulaciones apendiculares con OA $(11 / 36,30,6 \%)$ que aquellos de momentos posteriores a la ACM $(9 / 40,22,5 \%)$ (Tabla 4). En el caso de las regiones articulares, las frecuencias de OA son similares entre el bloque temporal pre ACM y post $\operatorname{ACM}(12 / 76,15,8 \%$ y 16/106, $15,1 \%$ respectivamente) (Tabla 4). Esto se ve respaldado a partir del test de $\mathrm{X}^{2}$, el cual no arrojó diferencias estadísticamente significativas entre bloques temporales ni para las articulaciones $\left(\mathrm{X}^{2}=1,27 ; \mathrm{gl}=1 ; \mathrm{p}=0,25\right)$ ni para las regiones articulares $\left(\mathrm{X}^{2}=0,01 ; \mathrm{gl}=1 ; \mathrm{p}=0,90\right)$ (Tabla 5$)$.

\section{Discusión}

La osteoartrosis es una patología con una etiología compleja debido a la multiplicidad de factores individuales y poblacionales que desencadenan los procesos fisiológicos que culminan en la afección de la articulación y en la producción de los rasgos morfológicos osteoartrósicos (Waldron 2009; Weiss y Jurmain 2007). Esta etiología multifactorial debe ser tomada en cuenta a la hora de interpretar la presencia de $\mathrm{OA}$ en el registro arqueológico en términos de actividades y patrones de uso del cuerpo. Sumado a ello, debe tenerse en cuenta el tamaño pequeño de la muestra disponible, que limita el alcance de las interpretaciones y el control de factores tales como sexo, edad, lateralidad y cronología, debido a que no admite demasiadas diferenciaciones internas en los análisis. Esta característica suele ser la norma en las series osteológicas derivadas de poblaciones cazadoras-recolectoras, pero es compensada aquí con la disponibilidad de información detallada sobre su contexto y de modelos arqueológicos explicativos para el área de estudio. De este modo, y pese a las limitaciones mencionadas, es posible identificar en la muestra patrones en los datos obtenidos sobre la frecuencia, distribución y severidad de la OA, que permiten discutir diversos posibles factores que los explican. A su vez, esto brinda la posibilidad de realizar 
una primera evaluación de las hipótesis planteadas sobre los patrones de actividad de los grupos humanos de los que deriva la serie osteológica del Lago Salitroso $\mathrm{y}$ trazar rumbos futuros en las investigaciones.

En este sentido, los resultados alcanzados hasta al momento, tanto en la columna vertebral como en las articulaciones apendiculares, muestran una frecuencia relativamente alta a nivel individual de alguna manifestación de OA, pero con frecuencias moderadas a bajas de afectación por articulación o superficie articular analizadas y con grados de severidad mayoritariamente leves.

Específicamente, la columna vertebral es el sector del esqueleto con mayores casos de OA. En ella, las regiones torácica y lumbar son las que presentan las mayores frecuencias de OA, seguidas por la región cervical. A su vez, se registró la existencia de variabilidad en el interior de las regiones afectadas, tal como se observa en la región torácica, en la cual el segmento inferior (T9-T12) presentó mayores prevalencias y grados más severos de OA que los segmentos, superior (T1-T4) y medio (T5-T8). Estos resultados son interesantes dado que el punto máximo de curvatura de la columna se localiza en el segmento medio de la región torácica (Bridges 1994; Brown et al. 2008; Merbs 1983), y, por lo tanto, es el que suele reflejar el estrés universal que experimenta la columna vertebral asociado al peso relativo que normalmente debe soportar este sector, la postura erguida y al bipedismo, y su acumulación en el tiempo (Adams et al. 1994; Knüsel et al. 1997). Asimismo, es esperable hallar bajas frecuencias de OA en T12 y L5/S1 debido a que se posicionan por detrás del centro de gravedad, y por lo tanto se ven relativamente menos involucradas en el soporte de peso (Bridges 1994; Brown et al. 2008). De esta forma, la bibliografía sugiere que la presencia de OA en las regiones torácica y lumbar refleja el estrés resultado de la carga, particularmente sobre la espalda, de pesos adicionales al peso normal que soporta la columna vertebral y trasmite a los miembros inferiores (Merbs 1983). Así, considerando los distintos tramos de la columna afectados, se sugiere que los resultados obtenidos podrían indicar que los individuos habrían realizado sus tareas cotidianas en una postura en la cual la columna vertebral se encontraba flexionada (p. ej., postura de sentado o arrodillado) (Adams y Hutton 1981; Bridges 1994; Brown et al. 2008; Capasso et al. 1999; Jurmain 1990; Knüsel et al. 1997; Lieverse et al. 2007; Lovell 1994; Sofaer Derevenski 2000; Swanepoel et al. 1995). Por lo tanto, se habrían visto comprometidas, particularmente, las regiones inferiores de la columna vertebral (T9-L5), provocando la deformación de los márgenes de los cuerpos vertebrales (Aufderheide y Rodríguez-Martín 1998; Bridges 1994; Burt et al. 2013; Waldron 2009). Por su parte, la baja frecuencia de OA en la región cervical, sugiere que estos individuos no realizaban de manera constante y repetida el levantamiento y traslado de cargas pesadas sobre la cabeza (Hukuda et al. 2000).

En lo que respecta al esqueleto apendicular, los resultados obtenidos mostraron que la pelvis es la articulación apendicular con mayor frecuencia de OA y la más severamente afectada, seguida por el hombro y la rodilla. Si bien diversos autores (Bridges 1992; Jurmain 1980, 1991; Weiss y Jurmain 2007) proponen que la presencia de osteoartrosis en las articulaciones apendiculares, particularmente el hombro y la pelvis, responden a la edad avanzada de los individuos, más que a los niveles de estrés mecánico y exigencia articular, sostienen que no es posible descartar la presencia de OA en las articulaciones apendiculares como resultado de las actividades físicas cotidianas. Sobre esta base, en primer lugar, la relativa elevada frecuencia de OA en la pelvis puede sugerir, por un lado, la ejecución de actividades tales como la movilidad, particularmente sobre superficies de terreno irregulares y duras, y por el otro, comportamientos relacionados con la carga y traslado de pesos sobre los miembros superiores, lo cual habría implicado una sobrecarga de dichas articulaciones (Assassi y Magnenat-Thalmann 2014; Bridges 1992; Capasso et al.1999; Henak et al. 2011; Jurmain 1977, 1980; Lieverse et al. 2007; Neves 1984; Quevedo Kawasaki 2000). En segundo lugar, la articulación del hombro participa en los movimientos de abducción o elevación del brazo, y en la flexión y extensión del mismo. Actividades tales como el lanzamiento de objetos, y la carga de pesos sobre los hombros, podrían contribuir a explicar la presencia de OA en dicha articulación (Capasso et al. 1999; Constantinescu 1999; Kennedy 1989; Rodrigues Carvalho 2004; Rodríguez Balboa et al. 2007; Rogers y Waldron 1995; Waldron 2009). Asimismo, las lesiones osteoartrósicas registradas en el hombro, podrían también estar vinculadas a la realización de movimientos en forma repetida y continuada, como, por ejemplo, las actividades de molienda, producción cerámica, preparación de cueros, entre otros (Jurmain 1977; Lieverse et al. 2007). Estas interpretaciones podrán evaluarse más adecuadamente en el futuro, incluyendo nuevos sistemas articulares no contemplados en esta etapa del estudio, tales como las articulaciones de la mano y la muñeca, las cuales habrían estado involucradas en la ejecución de dichas actividades.

Por último, como se mencionó, la rodilla, fue la articulación que registró la menor frecuencia de OA de las tres articulaciones apendiculares analizadas. La rodilla participa de la flexión y extensión de las piernas, a la vez que son fundamentales para sostener el peso corporal (Lieverse et al. 2007). Las modificaciones óseas en esta articulación podrían vincularse con la hiperextensión e hipermovilidad de la misma, como consecuencia del 
uso, que resulta en un aumento de la inestabilidad de la articulación y en una predisposición a la modificación mecánica (Aufderheide y Rodríguez-Martin 1998; Burt et al. 2013; Desloovere et al. 2010; Ortner 2003; Waldron 2009). Actividades tales como la deambulación, particularmente mientras se acarrean cargas pesadas (Aufderheide y Rodríguez-Martín 1998; Bridges 1991; Burt et al. 2013; Capasso et al.1999; Jurmain 1977, 1980; Lieverse et al. 2007; Neves 1984; Ortner 2003; Waldron 2009) y la ejecución de tareas en postura acuclillada de manera recurrente y durante prolongados periodos de tiempo pueden provocar lesiones osteoartrósicas en dicha articulación (Capasso et al. 1999; Kennedy 1989; Lieverse et al. 2007).

En relación con la bilateralidad, los resultados obtenidos indican, tanto a nivel de las articulaciones como de las regiones articulares, que el lado izquierdo presenta una mayor frecuencia de OA, particularmente a nivel de las articulaciones del hombro y la rodilla izquierdas. Contrariamente, la articulación de la pelvis presenta frecuencias de OA levemente mayores para el lado derecho. No obstante, dichas diferencias no fueron estadísticamente significativas y el tamaño de la muestra es aún pequeño, por lo que no puede descartarse que un aumento de casos analizados modifique la tendencia en cualquier dirección.

En el mismo sentido, la ausencia de diferencias estadísticamente significativas entre los sexos en los casos positivos de OA vertebral y apendicular sugieren que las actividades realizadas por ambos sexos habrían involucrado frecuencias e intensidades similares, lo que se plasmaría en niveles de exigencia articular similar, independientemente de las tareas puntuales asignadas a los sexos. Esto último se condice con lo postulado por Weiss (2005), quien sugiere la ausencia de una correlación fuerte entre el sexo y la osteoartrosis.

En cuanto a la edad, a pesar de que no se han hallado diferencias estadísticamente significativas entre grupos etarios en los casos positivos de OA, es destacable que las mayores frecuencias de afectación y mayor severidad se encuentran en los individuos mayores de 40 años, y probablemente sean producto del carácter acumulativo del desgaste fisiológico de las articulaciones, propio del avance de la edad y de la intervención de procesos sistémicos asociados al envejecimiento (Jurmain 1977; Weiss 2005; Zhang y Jordan 2010). De todos modos, se destaca la existencia de individuos menores a 40 años con casos positivos de OA, los cuales dan cuenta de la existencia de un compromiso corporal que podría ser el desencadenante del desarrollo de la osteoartrosis en la población de adultos jóvenes y medios de la colección.

Finalmente, en relación con los bloques temporales analizados, se observaron diferencias estadísticamente significativas en los casos de OA entre ellos, hallándose mayores frecuencias de $\mathrm{OA}$ en individuos de momentos pre ACM que en aquellos de momentos post ACM. En este sentido, por el momento se sugiere que estas diferencias podrían responder más a cuestiones de sesgos etarios de la muestra bajo estudio que a cambios en los patrones de movilidad o actividad. Como se mencionó, se ha detectado una diferencia en la composición etaria de las muestras integrantes de ambos bloques, dado que la muestra pre ACM cuenta con una mayor cantidad de individuos mayores a 40 años que aquella de momentos posteriores a la ACM. Será necesario incluir nuevos casos, aún a expensas de su completitud anatómica, para mejorar el balance etario de las muestras.

Como se mencionó anteriormente, con las limitaciones discutidas sobre el tamaño de la muestra y el uso de la OA como indicador de actividad, los resultados obtenidos permiten comenzar a discutir las hipótesis más generales planteadas a nivel poblacional. En los párrafos anteriores se mencionó como los patrones observados a nivel de cada articulación apendicular o de la columna vertebral podían vincularse, al menos parcialmente, con la actividad física repetida. En términos poblacionales, sobre la base de la información etnográfica y de diversas fuentes clásicas etnohistóricas disponibles, se ha sugerido que los grupos cazadores-recolectores patagónicos habrían tenido fuertes demandas físicas relacionadas con sus frecuentes desplazamientos residenciales en búsqueda de lugares óptimos para el asentamiento, en relación a la cercanía de recursos básicos (Aguerre 2000; Cox 1863; Escalada 1949; Lista 1879; Martinic 1995; Musters 1911; Schmidt 1964). Por su parte, a partir de la evidencia arqueológica recuperada para el área de estudio presentada aquí, se ha propuesto que los grupos humanos se habrían asentado en sectores con cuerpos de agua próximos, dado su rol como recurso crítico, y se habrían trasladado grandes distancias hacia sectores aledaños como las tierras altas (p.ej., Pampa del Asador) para el aprovisionamiento de materia prima lítica, dinámica que se habría acentuado en momentos posteriores a la ACM y habría involucrado una reducción de la movilidad residencial (Cassiodoro 2011; Cassiodoro et al. 2004; Goñi 2000; Goñi y Barrientos 2004; Goñi et al. 2000-2002). Estos traslados a lo largo de extensas porciones del terreno habrían implicado un retorno al asentamiento con carga de pesos, tal como el acarreo de personas (p.ej., niños sin capacidad de trasladarse por sus propios medios), de recursos animales y objetos pesados (p.ej., cueros, agua, leña, recursos animales y vegetales, materias primas líticas, entre otros) (Cox 1863; Lista 1879; Martinic 1995; Musters 1911; Schmidt 1964). La actividad deambulatoria con carga de bienes sobre el sector de los hombros y la espalda, pudo tener un alto impacto en las articulaciones de los miembros inferiores y de la columna vertebral, afectando principalmente al segmento inferior de la región torácica y a la región lumbar (Aufderheide y Rodríguez-Martín 1998; Bridges 1994; 
Lovell 1994; Ortner 2003; Quevedo Kawasaki 2000; Rodríguez Balboa et al. 2007). A su vez, la presencia de lesiones traumáticas (p.ej., espondilólisis de la quinta vértebra lumbar) y malformaciones congénitas (p.ej., presencia de sextas vértebras lumbares, malformaciones en la región cervical) (García Guraieb 2010) pueden favorecer la expresión de lesiones osteoartrósicas, ya que alteran las curvaturas normales de la columna, permiten reforzar esta idea.

Por otro lado, es importante destacar que, pese a que la información etnográfica y arqueológica disponibles indican que las poblaciones patagónicas del Holoceno Tardío habrían tenido requerimientos físicos importantes. Estos se manifiestan solo parcialmente en términos de frecuencia y, sobre todo de severidad, en las lesiones osteoartrósica en la muestra analizada. De hecho, los resultados obtenidos para la serie del Lago Salitroso contrastan en forma marcada con estudios realizados por otros investigadores en colecciones osteológicas de cazadores-recolectores, en los que se han hallado elevadas frecuencias de OA con grados severos, acompañada de otras artropatías tales como los nódulos de Schmorl, y la existencia de diferencias estadísticamente significativas entre los sexos (Flensborg 2012; Luna 2008; Mazza 2015; Rodríguez Balboa et al. 2007; Scabuzzo 2010; entre otros).

Diversas situaciones pueden explicar estas diferencias. En primer lugar, y como se ha mencionado ya, esto podría deberse, por un lado, a que se trata de una muestra pequeña con abundancia de individuos adultos jóvenes, y por el otro, a que la osteoartrosis no resulte un indicador adecuado o lo suficientemente sensible para abordar los niveles de actividad física, en particular en una colección con estas características. En este sentido, y en segundo lugar, otros indicadores, tales como los cambios entésicos y la robusticidad ósea, podrían resultar más adecuados para abordar el problema de la actividad física en esta particular colección osteológica. De esta forma, cabe mencionar la marcada robustez general ósea presente en esta colección, la cual resulta esperable dado que se trata de un grupo pedestre, con una fuerte demanda de movimientos asociados a este modo de vida. Sin embargo, es posible que dicha exigencia no llegue a impactar a nivel articular dado que la robusticidad muscular puede actuar como "protectora" de la articulación contra las lesiones y artropatías como la OA (Knüsel 1993; Weiss 2005). En relación con esto, al tratarse de grupos cazadores-recolectores pedestres, las actividades físicas de alta intensidad, particularmente, la movilidad a través de extensas porciones de terreno involucraría a todos los integrantes del grupo, propiciando el desarrollo muscular en individuos subadultos desde edades tempranas tanto prepuberales como en la adolescencia, lo que podría estar funcionando como una respuesta adaptativa contra la $\mathrm{OA}$.

\section{Conclusiones}

El estudio de la osteoartrosis como línea de evidencia para abordar las actividades físicas cotidianas y los niveles de demanda mecánica, y de esta forma conocer algunos de los aspectos del estilo de vida, particularmente aquellos relacionados al uso del cuerpo, constituye uno de los aspectos más recurrentemente desarrollados en la Antropología Biológica y la Bioarqueología a escala mundial. Es en este sentido que se formularon una serie de expectativas en torno a los patrones generales de actividad corporal y de la organización de las actividades entre los individuos integrantes de los grupos cazadoresrecolectores de Patagonia Centro-meridional durante el Holoceno Tardío. En esta instancia, se presenta aquí el primer estudio sistemático de la presencia de $\mathrm{OA}$ en individuos adultos de la cuenca del Lago Salitroso, cuyo enfoque estuvo basado no solo en un relevamiento paleopatológico de la condición sino también en la exploración y discusión de su potencial utilidad como indicador del uso del cuerpo y del estrés mecánico al que estuvieron sujetos los individuos integrantes de la población bajo estudio. A su vez, se empleó la información recuperada por otras líneas de investigación arqueológica, fuentes etnohistóricas y relatos de viajeros, como base para llevar adelante la discusión de la interpretación de dichas modificaciones óseas en términos del comportamiento y compromiso corporal.

La información obtenida a partir de la evaluación de esta patología, ilustra claramente la necesidad de realizar investigaciones futuras sobre las lesiones osteoartósicas. De esta forma, es necesario ampliar el tamaño de la muestra, tanto a nivel de la cantidad de individuos como de la cantidad de articulaciones y regiones articulares analizadas, particularmente introducir articulaciones más pequeñas, tales como muñeca/mano y tobillo/pie, y considerar esqueletos con menores grados de integridad anatómica de la colección. De igual forma, resulta necesario introducir nuevas líneas de investigación como el relevamiento de cambios entésicos y la robusticidad ósea, preferentemente a través de métodos de diagnóstico por imágenes, como tomografías computadas, que permitan ampliar la base de los indicadores óseos de actividad física empleados.

En suma, los resultados obtenidos aquí vienen a llenar un vacío de información sobre la OA en la serie del Lago Salitroso, arrojando las primeras luces en la discusión sobre su patrón paleoepidemiológico, el estrés mecánico al que estuvieron sujetas las poblaciones patagónicas y los cambios en diferentes aspectos de la organización de sus actividades registrados durante los últimos milenios del Holoceno Tardío (para mayor detalle ver Morlesín 2019). Sobre esta base, se articularán los nuevos caminos de investigación a desarrollar. 
Agradecimientos: Este trabajo fue realizado a partir de la colección osteológica recuperada a través de las investigaciones realizadas bajo los subsidios UBACYT dirigidos por el Dr. Rafael Goñi: F072 (2001- 2003),
F065 (2004-2007), F031 (2008-2010), 20020100100441 (2011-2014), 20020130100293BA (2014-2017). Agradecemos a los evaluadores de este trabajo por sus útiles comentarios y sugerencias.

\section{Referencias Citadas}

Adams, M. y W. Hutton 1981. The relevance of torsion to the mechanical derangement of the lumbar spine. Spine 6:241-248.

Adams, M., D. McNally, H. Chinn y P. Dolan 1994. Posture and the compressive strength of the lumbar spine. Clinical Biomechanics 9:5-14.

Aguerre, A. 2000. Las vidas de Pati en la Toldería Tehuelche del Rio Pinturas y el Después. Facultad de Filosofía y Letras, Universidad de Buenos Aires, Buenos Aires.

Arden, N. y M. Nevitt 2006. Osteoarthritis: Epidemiology. Best practice and research. Clinical Rheumatology 20 (1):03-25.

Arencibia, V., C. Crespo, S. García Guraieb, M. Russo, C. Dejean y R. Goñi 2019. Análisis genético poblacional de grupos cazadoresrecoltores del Holoceno tardío del lago Salitroso (Santa Cruz, Argentina). Revista Argentina de Antropología Biológica 21 (2):1-16.

Arrieta, M. y O. Mendonça 2011. Enfermedad degenerativa articular y uso del cuerpo en Rincón Chico 21. Revista Argentina de Antropología Biológica 13:03-17.

Assassi, L. y N. Magnenat-Thalmann 2014. A biomechanical approach for dynamic hip joint analysis. En 3D Multiscale Physiological Human, editado por N. Magnenat-Thalmann, O. Ratib y H. Choi, pp. 233-252. Springer, Londres.

Aufderheide, A. y C. Rodríguez-Martín 1998. The Cambridge Encyclopedia of Human Paleopathology. Cambridge University Press, Cambridge.

Bernal, V., S. García Guraieb y P. González 2004. Perfiles de mortalidad de las muestras de restos óseos humanos procedentes del área del lago Salitroso (provincia de santa Cruz). En Contra Viento y Marea. Arqueología de Patagonia, editado por T. Civalero, P. Fernández, y G. Guraieb, pp. 361-373. INAPL-SAA, Buenos Aires.

Binford, L. 2001. Constructing Frames of Reference. An Analytical Method for Archaeological Theory Building Using Ethnographic and Environmental Data Sets. University of California Press, Berkeley.

Bridges, P. 1991. Degenerative joint disease in hunter-gatherers and agriculturalists from the southeastern USA. American Journal of Physical Anthropology 85:379-391.

Bridges, P. 1992. Prehistoric arthritis in the americas. Annual Review of Anthropology 21:67-91.

Bridges, P. 1993. The effect of variation in methodology on the outcome of osteoarthritic studies. International Journal of Osteoarchaeology 3:289-295.

Bridges, P. 1994. Vertebral arthritis and physical activities in the prehistoric southeastern USA. American Journal of Physical Anthropology 93:83-93.

Brown, K., P. Pollintine y M. Adams 2008. Biomechanical implications of degenerative joint disease in the apophyseal joints of human thoracic and lumbar vertebrae. American Journal of Physical Anthropology 136:318-326.

Burt, N., D. Semple, K. Waterhouse y N. Lovell 2013. Identification and Interpretation of Joint Disease in Paleopathology and Forensic Anthropology. Charles C Thomas Publisher, LTD, Springfield, Illinois.
Capasso, L., K. Kennedy y C. Wilczak 1999. Atlas of Occupational Markers on Human Remains. Edigrafital S.P.A., Teramo.

Carmona Ortells, L. 2010. Epidemiología de la artrosis. En Artrosis. Fisiopatología, Diagnóstico y Tratamiento, editado por J. Monfort, pp. 3-17. Sociedad Española de Reumatología y Editorial Médica Panamericana, Madrid.

Cassiodoro, G. 2011. Movilidad y Uso del Espacio de Cazadores Recolectores del Holoceno Tardío. Estudio de la Variabilidad del Registro Tecnológico en Distintos Ambientes del Noroeste de la Provincia de Santa Cruz (Argentina). BAR 2259, Archaeopress, Oxford.

Cassiodoro, G. y S. García Guraieb 2009. Análisis del registro tecnológico y osteológico de los entierros humanos del Holoceno tardío del lago Salitroso (Santa Cruz). En Arqueología de Patagonia: una Mirada desde El Último Confín, editado por M. Salemme, F. Santiago, M. Álvarez, E. Piana, M. Vázquez y E. Mansur, Tomo II, pp. 613-628. Editorial Utopías, Ushuaia.

Cassiodoro, G., G. Guráieb, A. Re y A. Tívoli 2004. Distribución de recursos líticos en sitios de superficie de la cuenca de los lagos Pueyrredón-Posadas-Salitroso. En Contra Viento y Marea. Arqueología de Patagonia, editado por T. Civalero, P. Fernández, y G. Guraieb, pp. 57-69. INAPL-SAA, Buenos Aires.

Constantinescu, F. 1999. Evidencias bioantropológicas para modos de vida cazador-recolector terrestre y marítimo en los restos óseos humanos de Tierra del Fuego. Anales del Instituto de la Patagonia 27:137-174.

Cox, G. 1863. Viaje en las Regiones Septentrionales de la Patagonia. Imprenta Nacional, Santiago.

Desloovere, K., P. Wong, L. Swings, B. Gallewaert, H. Vandenneucker y A. Leardini 2010. Range of motion and repeatability of knee kinematics for 11 clinically relevant. motor tasks. Gait \& Posture 32:597-602.

Escalada, F. 1949. El Complejo “Tehuelche”. Estudios de Etnografía Patagónica. Instituto Superior de Estudios Patagónicos, Buenos Aires.

Flensborg, G. 2012. Análisis Paleopatológico en el Curso Inferior del Río Colorado (Pcia. de Buenos Aires). Exploración y Evaluación del Estado de Salud de Sociedades Cazadoras-Recolectoras en el Holoceno Tardio. Tesis Doctoral. Facultad de Ciencias Sociales, Universidad Nacional del Centro de la Provincia de Buenos Aires, Olavarría.

García Guraieb, S. 2006. Salud y enfermedad en cazadoresrecolectores del Holoceno tardío en la cuenca del lago Salitroso (Santa Cruz). Intersecciones en Antropología 7:37-48.

García Guraieb, S. 2010. Bioarqueología de Cazadores-Recolectores del Holoceno Tardio de la Cuenca del Lago Salitroso (Santa Cruz): Aspectos Paleopatológicos y Paleodemográficos. Tesis Doctoral. Facultad de Filosofía y Letras, Universidad de Buenos Aires, Buenos Aires.

García Guraieb, S., P. González y V. Bernal 2007. Estructura de sexo y edad de la muestra de restos humanos del Holoceno tardío del lago Salitroso (Santa Cruz, Argentina). En Arqueología de Fuego-Patagonia. Levantando Piedras, Desenterrando Huesos 
y Develando Arcanos, editado por F. Morello, M. Martinic, A Prieto y G. Bahamonde, pp. 367-374. Ediciones CEQUA, Punta Arenas.

García Guraieb, S., R. Goñi y R. Guichón Fernández 2018. Aporte a la cronología de chenques tardíos del lago Salitroso (Santa Cruz, Argentina). Revista Arqueología 24:271-280.

García Guraieb, S., R. Goñi y A. Tessone 2015. Paleodemography of late Holocene hunter-gatherers from Patagonia (Santa Cruz, Argentina): an approach using multiple archaeological and bioarchaeological indicators. Quaternary International $365: 147-158$

García Guraieb, S. y M. Maldonado 2014. Salud Bucal en Grupos Cazadores-Recolectores Patagónicos del Holoceno tardío del lago Salitroso (Santa Cruz, Argentina). En Avances Recientes de la Bioarqueología Latinoamericana, editado por L. Luna, C. Aranda y J. Suby, pp. 231-254. Grupo de Investigación en Bioarqueología, Buenos Aires.

Goñi, R. 1991. Estrategias adaptativas de momentos tardíos en el PNPM (Santa Cruz, Argentina). Resúmenes de Simposios del XII Congreso Nacional de Arqueología Chilena, pp. 4-5. Museo Regional de la Araucanía - Sociedad Chilena de Arqueología Dirección General de Bibliotecas, Archivos y Museos, Temuco.

Goñi, R. 2000. Arqueología de Momentos Históricos fuera de los centros de conquista y colonización: un análisis de caso en el sur de la Patagonia. En Desde el País de los Gigantes. Perspectivas Arqueológicas en Patagonia. Tomo 1, pp. 283-296. Universidad Nacional de la Patagonia Austral, Río Gallegos.

Goñi, R. 2010. Cambio Climático y Poblamiento Humano durante el Holoceno Tardío en Patagonia Meridional. Una Perspectiva Arqueológica. Tesis Doctoral. Facultad de Filosofía y Letras, Universidad de Buenos Aires, Buenos Aires.

Goñi, R. y G. Barrientos 2004. Poblamiento tardío y movilidad en la cuenca del lago Salitroso. En Contra Viento y Marea. Arqueología de Patagonia, editado por T. Civalero, P. Fernández, y G. Guraieb, pp. 313-324. INAPL-SAA, Buenos Aires.

Goñi R., S. Barrientos y G. Cassiodoro 2000-2002. Condiciones previas a la extinción de las poblaciones humanas del sur de Patagonia: una discusión a partir del análisis del registro arqueológico de la cuenca del Lago Salitroso. Cuadernos del Instituto Nacional de Antropología Pensamiento Latinoamericano 19:249-266.

Goñi, R., L. Bosio y S. García Guraieb 2003-2005. Un caso de enfermedad infecciosa en cazadores recolectores prehispánicos de Patagonia. Cuadernos del Instituto Nacional de Antropología y Pensamiento Latinoamericano 20:399-404.

Guichón Fernández, R. 2016. Estudio de Individuos Subadultos de la Cuenca del Lago Salitroso. Evaluación de Sesgos en la Representación Etaria en Chenques del Holoceno Tardío Final (Santa Cruz). Tesis de Licenciatura. Facultad de Ciencias Sociales, Universidad Nacional del Centro de la provincia de Buenos Aires, Buenos Aires.

Henak, C., B. Ellis, M. Harris, A. Anderson, C. Peters y J. Weiss 2011. Role of the acetabular labrum in load support across the hip joint. Journal of Biomechanics 44:2201-2206

Hooper, L., K. Demps, M. Gurven, D. Gerkey y H. Kaplan 2015. Skills, division of labour and economies of scale among Amazonian hunters and South Indian honey collectors. Philosophical Transaction Royal Society B 370:1-11.

Hukuda, S., K. Inoue, T. Ushiyama, Y. Saruhashi, A. Iwasaki, J. Huang, A. Mayeda, M, Nakai, F. Xiang Li y Z. Qing Yang 2000
Spinal degenerative lesions and spinal ligamentous ossifications in ancient chinese populations of the yellow river civilization. International Journal of Osteoarchaeology 10:108-124.

Hunter, D. 2011. Osteoarthritis. Best Practice and Research Clinical Rheumatology 25 (6):801-814.

Jurmain, R. 1977. Stress and the etiology of osteoarthritis. American Journal of Physical Anthropology 46:353-366.

Jurmain, R. 1980. The pattern of involvement of appendicular degenerative joint disease. American Journal of Physical Anthropology $53: 143-150$

Jurmain, R. 1990. Paleoepidemiology of a central California prehistoric population from CA-ALA-329: II. Degenerative Disease. American Journal of Physical Anthropology 83:83-94.

Jurmain, R. 1991. Degenerative changes in peripheral joints as indicators of mechanical stress: opportunities and limitations. International Journal of Osteoarchaeology 1:247-252.

Kennedy, K. 1989. Skeletal markers of occupational stress. En Reconstruction of Life from the Skeleton, editado por M.Y. Işcan y K. Kennedy, pp. 129-160. Alan R. Liss, Nueva York.

Knüsel, C. 1993. On the biomechanical and osteoarthritic differences between hunter-gatherers and agriculturalists. American Journal of Physical Anthropology 91:523-527.

Knüsel, C., S. Gögel y D. Lucy 1997. Comparative degenerative joint disease of the vertebral column in the medieval monastic cemetery of the Gilbertine Priory of St. Andrew, York, England. American Journal of Physical Anthropology 103:481-495.

Landis, J. y G. Koch 1977. The measurement of observer agreement for categorical data. Biometrics 33 (1):159-174.

Larsen, C. 1995. Prehistoric human biology of the Carson desert: a bioarchaeological investigation of a hunter-gatherer lifeway. En Bioarchaeology of the Stillwater Marsh: Prehistoric Human Adaptation in the Western Great Basin, editado por C. Larsen y R. Kelly, pp. 33-40. Anthropological Papers of the American Museum of Natural History, Washington.

Larsen, C. 1997. Bioarchaeology: Interpreting Behavior from the Human Skeleton. Cambridge University Press, Cambridge.

Lieverse A., A. Weber, V. Bazaliiskiy, O. Goriunova y N. Savel'ev 2007. Osteoarthritis in Siberia's Cis-Baikal: skeletal indicators of hunter-gatherer adapatation and cultural change. American Journal of Physical Anthropology 132:1-16.

Lieverse, A., B. Mack, V. Bazaliiskiy y A. Weber 2016. Revisiting osteoarthritis in the Cis-Baikal: understanding behavioral variability and adaptation among middle Holocene foragers. Quaternary International 405 (B):160-171.

Lista, R. 1879. Viaje al País de los Tehuelches. Exploraciones en la Patagonia Austral. Imprenta M. Biedma, Buenos Aires.

Lovell, N. 1994. Spinal arthritis and physical stress at Bronze Age Harappa. American Journal of Physical Anthropology 93:149-164.

Luna, L. 2008. Estructura Demográfica, Estilo de Vida y Relaciones Biológicas de Cazadores Recolectores en un Ambiente de Desierto. Sitio Chenque I (Parque Nacional Lihué Calel, Provincia de la Pampa, Argentina). BAR 1886, Archaeopress, Oxford.

Luna, L., C. Aranda y A. Amorim Alves 2017. Reflexiones sobre el relevamiento y análisis comparativo de patologías osteoarticulares en restos esqueletales humanos. Revista Argentina de Antropología Biológica 19 (1):1-8.

Martinic, M. 1995. Patagonia de Ayer y de Hoy. Talleres Gráficos Juan Buvinic, Punta Arenas. 
Mazza, B. 2015. Estudio de los Patrones de Variación Morfológica en Restos Humanos del Humedal del Paraná inferior. Tesis Doctoral. Facultad de Filosofía y Letras, Universidad de Buenos Aires, Buenos Aires.

Merbs, C. 1983. Patterns of Activity-induced Pathology in a Canadian Inuit Population. National Museum of Man Mercury Series, Ottawa.

Morlesín, M. 2019. Patrones de Actividad y Organización Social de los Grupos Cazadores-Recolectores de la Cuenca del Lago Salitroso, Provincial de Santa Cruz. (Argentina) durante el Holoceno Tardío: Un Estudio de las Modificaciones Osteológicas (Artropatías). Tesis de Licenciatura. Facultad de Filosofía y Letras, Universidad de Buenos Aires, Buenos Aires.

Musters, G. 1911. Vida entre los Patagones. Universidad Nacional de La Plata, Buenos Aires.

Neves, W. 1984. Estilo de vida e osteobiografía: a reconstituiçao do comportamento pelos ossos humanos. Revista de Pré-historia VI:287-291.

Ortner, D. 2003. Identification of Pathological Conditions in Human Skeletal Remains. Academic Press, San Diego.

Quevedo Kawasaki, S. 2000. Patrones de actividad a través de las patologías en población arcaica de Punta Teatinos, norte semiárico chileno. Chungara Revista de Antropología Chilena 32 (1):11-21.

Resnick, D. 2002. Degenerative disease of the spine. En Diagnosis of Bone and Joint Disorders, editado por D. Resnick, pp.13821485. WB Saunders, Philadelphia.

Rodrigues Carvalho, C. 2004. Marcadores de Estresse Ocupacional em Populações Sambaquieiras do Litoral Fluminense. Tesis Doctoral. Fundación Osvaldo Cruz, Rio de Janeiro.

Rodríguez Balboa, M., E. Aspillaga Fontaine y B. Arensburg 2007. El estudio bioantropológico de las colecciones esqueletales del archipiélago de Chiloé: perspectivas y limitaciones. En Arqueología de Fuego-Patagonia. Levantando Piedras, Desenterrando Huesos... y Develando Arcanos, editado por F. Morello, M. Martinic, A. Prieto y G. Bahamonde, pp. 269-278. CEQUA, Punta Arenas.

Rogers, J. y T. Waldron 1995. A Field Guide to Joint Disease in Archaeology. John Wiley \& Sons, Chichester.
Rogers, J., T. Waldron, P. Dieppe y I. Watt 1987. Arthropathies in palaeopathology: The basis of classification according to most probable cause. Journal of Archaeological Science 14:179-193.

Rojas-Sepúlveda, C., Y. Ardagna y O. Dutour 2008. Paleoepidemiology of vertebral degenerative disease in pre-columbian Muisca series from Colombia. American Journal of Physical Anthropology 135:416-430.

Scabuzzo, C. 2010. Actividad, Patología y Nutrición de los Cazadores-Recolectores Pampeanos. Tesis Doctoral. Facultad de Ciencias Naturales y Museo, Universidad Nacional de La Plata, Buenos Aires.

Schmidt, T. 1964. Misionando por Patagonia Austral. Academia Nacional de la Historia, Buenos Aires.

Sofaer Derevenski, J. 2000. Sex differences in activity-related osseous change. American Journal of Physical Anthropology 111:333-354.

Stine, S. 1994. Extreme and persistent drought in California and Patagonia during mediaeval time. Nature 369:546-549.

Swanepoel, M., L. Adams y J. Smeathers 1995. Human lumbar apophyseal joint damage and intervertebral disc degeneration. Annals of the Rheumatic Diseases 54:182-188.

Tessone, A., S. García Guraieb, R. Goñi y H. Panarello 2015. Isotopic evidence of weaning in hunter-gatherers from the late holocene in Lake Salitroso, Patagonia, Argentina. American Journal of Physical Anthropology 158:105-115.

Waldron, T. 2009. Paleopathology. Cambridge University Press, Cambridge.

Weiss, E. 2005. Understanding osteoarthritis patterns: an examination of aggregate osteoarthritis. Journal of Paleopathology 16 (1):87-98.

Weiss, E. y R. Jurmain 2007. Osteoarthritis revisited: a contemporary review of aetiology. International Journal of Osteoarchaeology $17: 437-450$.

Zhang, Y. y J. Jordan 2010. Epidemiology of osteoarthritis. Clinics in Geriatric Medicine 26 (3):355-369.

\section{Notas}

${ }^{1}$ Siguiendo a Luna et al. (2017), los términos OA y EDA pueden ser utilizados como sinónimos ya que dan cuenta del componente degenerativo de la articulación y el carácter proliferativo de la patología. Se utiliza el término OA dado que consideramos es más adecuado para describir e interpretar las manifestaciones patológicas articulares, ya que es un derivado de una enfermedad crónica degenerativa (Luna et al. 2017).

${ }^{2}$ Los nichos son oquedades rocosas naturales poco profundas (aproximadamente 1,5 m) donde los cuerpos se depositaron y cubrieron total o parcialmente por la sedimentación natural. En los EBB se aprovecha algún rasgo natural del terreno, como bloques erráticos o el filo de un afloramiento rocoso, en la construcción de las estructuras de rocas. Los chenques son estructuras de piedra acumuladas intencionalmente situadas sobre y alrededor de los cuerpos, que presentan una morfología de contorno oval, elíptico o circular, con dimensiones de entre de 3 a 5 metros tanto de largo como de ancho (García Guraieb 2010; Goñi y Barrientos 2004; Goñi et al. 2000-2002). 
